\title{
3 Research Square

\section{Generation of New Salt-Tolerant Wheat Lines and Transcriptomic Exploration of the Responsive Genes to Ethylene and Salt Stress}

\section{Qian Ma}

Qingdao Agricultural University

Huajian Zhou

Qingdao Agricultural University

\section{Xinying Sui}

Qingdao Agricultural University

\section{Chunxue Su}

Qingdao Agricultural University

\section{Yanchong Yu}

Qingdao Agricultural University

\section{Hongbing Yang}

Qingdao Agricultural University

Chun-Hai Dong ( $\nabla$ chunhai79@yahoo.com )

Qingdao Agricultural University https://orcid.org/0000-0003-0568-0300

\section{Research article}

Keywords: wheat, salt, ethylene sensitivity, mutagenesis, transcriptome, ERFs

Posted Date: July 27th, 2020

DOl: https://doi.org/10.21203/rs.3.rs-42817/v1

License: (c) (1) This work is licensed under a Creative Commons Attribution 4.0 International License.

Read Full License

Version of Record: A version of this preprint was published at Plant Growth Regulation on February 3rd, 2021. See the published version at https://doi.org/10.1007/s10725-021-00694-9. 


\section{Abstract}

Background: Wheat (Triticum aestivum L.) is a staple crop in the world, but is only moderately salt tolerant. However, salt stress affects one-fifth of irrigated agricultural land in the world, it is of great importance to cultivate salt-tolerant varieties to improve the global wheat production.

Results: In this study, over 90,000 wheat seeds of cultivar 'Luyuan502' were mutated by EMS, and 2000 salt-tolerant lines were harvested from salinized field. By analysis of ethylene sensitivity, salt related physiological factors, and preliminary crop yield, 12 salt-tolerant wheat lines with high production were selected among the crop plants. Transcriptome analysis indicated that a large number of the transcripts levels were significantly altered, mainly based on antenna proteins involved in photosynthesis, biosynthesis of secondary metabolites, cyanoamino acid metabolism, carotenoid biosynthesis, thiamine metabolism, and cutin, suberine and wax biosynthesis pathways including CABs, PERs/PODs, BGLUs, CYP707s, and ZEPS. qRT-PCR analysis revealed that the expressions of salt-related genes in the wheat lines were mostly higher than the wild type, and salt stress can significantly increase the expression levels of the ethylene-related genes in the wheat lines. Based on transcriptomic data, nine novel wheat ERFs were identified and analyzed, and it is suggested that they may play important roles in mediation of ethylene response and salt tolerance.

Conclusion: Salt-tolerant wheat mutant lines with ethylene insensitivity were obtained from screen of a wheat EMS-mutagenized pool. Transcriptome data showed that the mutant plants exhibit significant alterations in the antenna proteins involved in various biological processes. Expression analysis suggests that ERFs may mediate ethylene response and salt tolerance of the wheat lines.

\section{Background}

Bread wheat (Triticum aestivum L., genome AABBDD) is a relatively young species originated from Middle East nearly 10,000 years ago. This hexaploid wheat was hybridized by domesticated allotetraploid wheat (Triticum turgidum L., genome BBAA) and diploid goat grass (Aegilops tauschii L., genome DD) [1, 2]. The higher ploidal level and complex genetic background of hexaploid wheat improved its adaptability to diverse environment and accelerated its spread in the world [1,3]. Today, bread wheat is one of the most important world crops with about 220 million ha planting area in the world, feeding one-third of the global population, and proving one-fifth of the global caloric requirements and more than half of the global carbohydrate requirements $[4,5]$.

Although hexaploid wheat has improved adaptability to diverse environment, it still frequently challenged by unfavorable environmental changes. Salt is one of the environmental stresses, and causes dramatic wheat yield reduction and quality loss due to the moderate tolerance of bread wheat to salt stress [6]. Soil salinity affects one-fifth of irrigated agricultural land in the world, and continues to aggregate due to the increasing population and deterioration of environment [7]. 
It was reported that salt accumulation induces osmotic stress and excess $\mathrm{Na}^{+}$that causes chloroplast damage, photosynthesis and respiration decrease, photorespiration increase, plant growth and production inhibition, root growth repression, and reactive oxygen species (ROS) accumulation [8-11]. Plant also evolves responding mechanisms to alleviate these negative effects. Such as, the expression of genes including superoxide dismutase (SOD), peroxidase (POD), malonic dialdehyde (MDA), and catalase (CAT), is activated to alleviate ROS induced oxidative damage in salt stress [12]. The $\mathrm{Na}^{+}$concentration is kept to a low level by $\mathrm{Na}^{+}$exclusion and compartmentalization to protect the $\mathrm{K}^{+}$uptake, cytosolic charge balancing, enzyme activation, and maintenance of cell turgor $[13,14]$. As a result, the salt tolerant plants resume growing at a reduced level, in which the plant hormones play important roles.

Plant hormones including auxin (AUX), gibberellin (GA), cytokinin (CK), abscisic acid (ABA), ethylene (ET), brassinosteroid (BR), jasmonate (JA), salicylic acid (SA), and strigolactone (SL), are all small mobile chemical compounds, which play important roles in plant growth, development, biotic resistance, and abiotic resistance [15-17]. It is believed that the plant hormones JA, SA, ABA, BR, and ET play crucial roles in biotic and abiotic stresses, which include salt stress [18]. For example, it was reported that salt induced $A B A$ accumulation, and $A B A$ treatment improved salt tolerance in plants $[19,20]$. Overexpression of the $A B A$ biosynthesis related genes including 9-cis-epoxycaroteniod dioxygenase gene (NCED) and low expression of osmotically responsive gene (LOS5) increased ABA concentration and improved salt tolerance [21-23].

Although ethylene is also known in regulating salt tolerance in plants, the functions of ethylene in salt tolerance in dicotyledonous plants and monocotyledonous plants are totally different [24, 25]. In dicotyledonous plant Arabidopsis, ethylene treatment increased salt tolerance, and the ethylene overproducer 1 (eto 1) mutant caused ethylene accumulation and promoted salt tolerance by reducing $\mathrm{Na}^{+}$ delivery from root to shoot [26, 27]. In plants, ethylene sensitivity is closed related with salt tolerance. For instance, the ethylene insensitive mutants ethylene response1-1 (etr1-1), ethylene insensitive4-1 (ein4-1), etr2-1, ein2-1, ein2-5, and ein3-1 showed increased salt sensitivity, while ethylene sensitive or oversensitive mutants etr1-7, constitutive triple respnse1-1 (ctr1-1), and EIN3 binding F-box1-1 (ebf1-1) ebf2-1 had increased salt tolerance [28-32]. These results indicated that ethylene treatment, production, and signaling positively improve salt tolerance in dicotyledonous plants. However, in monocotyledonous plant rice, lack of MHZ6/OSEIN3-LIKE1 (OSEIL 1) and OSEIL2 caused ethylene insensitive and salt tolerance improvement, but their overexpression lines showed ethylene over-sensitivity and decreased salt tolerance through regulation of $\mathrm{K}^{+}$transport and $\mathrm{Na}^{+}$uptake in the rice seedling roots [33]. However, less is known about the regulatory functions of ethylene signaling in salt resistance of wheat plants. This study provides clues suggesting that the ethylene sensitivity is closely related with salt tolerance in wheat, and the mutant lines with decreased ethylene sensitivity exhibit enhanced salt tolerance.

Considering the importance of bread wheat for food supplement in the world and the deterioration of continuous soil salinity, there is an urgent need to improve the salt tolerance of bread wheat to meet future global food requirements. In this study, the wheat seeds of cultivar 'Luyuan502' were mutated by 
ethylmethylsulfonate (EMS), new salt-tolerant wheat lines were screened according to their ethylene sensitivity and physiological detection, and their stress resistance was examined by salt related gene expression and transcriptomic analysis.

\section{Results}

\section{Generation and development of a new salt-tolerant wheat library}

In order to achieve a new salt-tolerant bread wheat lines to satisfy future global food requirements, the bread wheat cultivar 'Luyuan502' was mutagenized by chemical mutagen EMS in this research. Firslty, about 500 seeds of cultivar 'Luyuan 502 ' were mutagenized by $0 \%, 0.2 \%, 0.4 \%, 0.6 \%, 0.8 \%$, and $1.0 \%$ EMS, respectively. The EMS-mutagenized seeds were sowed, germinated, and grown in a saline-alkali soil $(0.3 \%$ - $0.7 \%$ salinity) located at Huanghe river delta (Maotuo experimental field, Lijin, Shandong province, China). After 3 times repeats, the results indicated that the germination rate of $0 \%, 0.2 \%, 0.4 \%$, and $0.6 \%$ EMS mutagenized bread wheat seeds were about $71 \%, 69 \%, 58 \%$, and $55 \%$ in the saline soil

(Supplementary material 1 ). The surviving rate was nearly $0 \%$ when the EMS concentration was more than $0.8 \%$ (Supplementary material 1 ). Although the germination rate of $0.6 \%$ EMS treatment was not obviously lower than $0.4 \%$ EMS treatment, the germination uniformity was significantly decreased at $0.6 \%$ EMS treatment. In order to obtain more mutated seedlings, $0.4 \%$ EMS was chosen as the optimal concentration for mutagenesis. In the end, over 90,000 seeds of bread wheat cultivar 'luyuan 502' were mutagenized and sowed in the salinity field.

Visible plant phenotypes were observed from the mutant population, expanding wheat germplasm resources. A large number of individuals from the $M_{2}$ population were observed to display visual phenotypes. The variant phenotypes observed in the field were divided into several categories: leaf phenotype, plant height, tiller numbers, maturity, seed coat color. Among them, a seed coat color mutant line can keep the phenotype from $\mathrm{M}_{2}$ to $\mathrm{M}_{3}$ and $\mathrm{M}_{4}$, indicating that mutation might occurred in a dominant gene for seed coat development.

In order to develop a salt-tolerant wheat library, about 90,000 EMS-mutagenized wheat seeds were planted in the salinized soil (soil salinity is about $0.3 \%-0.7 \%$ ) of Maotuo experimental field, Lijin County, Shandong Province, China. About 60,000 wheat M1 plants were obtained. Among them, about 2000 M1 plants showing strong salt tolerance and good growth in the saline soil (Fig. 1A - D) were harvested. A single-seed descent population was developed to screen for salt tolerance.

To screen for high yield wheat lines from the salt-tolerant plants, the new salt-tolerant wheat lines and their wild type control 'Luyuan502' were planted in same quantity $(150 \mathrm{~g})$ and same area $\left(20 \mathrm{~m}^{2}\right)$ in the saline soil in Maotuo experimental field (Fig. 1E-H). According to the seeds harvested from $\mathrm{M}_{3}$ generation, 11 new salt-tolerant wheat lines with high yield were selected, and the results were summarized in the Table 1. In details, the results indicated that the productions of new salt-tolerant lines 
$06-6,1-13,1-29,1-105,1-15,2-7$, and $1-21$ increased about $20 \%-30 \%$ compared with wild type, and the productions of $1-119,1-18,1-98$, and $1-14$ increased about $10 \%-20 \%$.

Table 1

The grain yield of new salt-tolerant wheat lines $\left(\mathrm{kg} / 666.7 \mathrm{~m}^{2}\right)$

\begin{tabular}{|llll|}
\hline Line \# & M3 (Lijin, 2018) & M4 (Wudi, 2019) & M4 (Yanzhou, 2019) \\
\hline WT & 354 & 338 & 322 \\
$06133-6$ & 462 & 419 & 324 \\
$1-13$ & 456 & 341 & 375 \\
$1-29$ & 441 & 396 & 348 \\
$1-105$ & 435 & 311 & 349 \\
$1-15$ & 432 & 369 & 344 \\
$2-7$ & 408 & 439 & 409 \\
$1-21$ & 405 & 377 & 326 \\
$1-119$ & 393 & 332 & 417 \\
$1-18$ & 387 & 354 & 330 \\
$1-98$ & 387 & 395 & 345 \\
$1-14$ & 381 & 437 & 345 \\
\hline
\end{tabular}

All M3 seeds were collected from the 2,000 independent M2 plants. To further examine the salt tolerance of the wheat lines, the M3 seeds were soaked and cultivated in the petri dish with $0,50,100,200,300 \mathrm{mM}$ $\mathrm{NaCl}$ solution for $48 \mathrm{~h}$, and the root length was measured and compared. The results indicated that the root length was getting shorter with the increase of $\mathrm{NaCl}$ concentration, and $150 \mathrm{mM} \mathrm{NaCl}$ was selected as the optimal concentration for the salt tolerant screening. As a result, 430 salt tolerant lines including 1-29, 2-7, 1-98, 1-105, and 1-119 showed longer root length than wild type after salt treatment (Fig. 2AC). The root growth phenotype of the salt tolerant lines were also examined by treatment with $0,100,200$, 300 and 400 mM D-mannitol, and the results indicated that the wheat lines were also tolerant to the osmotic stress (Fig. 2D - F).

\section{Screen for the wheat mutant lines with alteration of ethylene sensitivity and salt resistance}

As ethylene insensitivity is highly related with stress tolerance in plants [28-32], the ethylene sensitivity of 430 new mutagenized wheat lines by salt screening were examined in this study. The salt-tolerant wheat seeds were soaked and cultivated in the petri dish supplemented with ethylene precursor 1aminocyclopropane-1-carboxylate (ACC) at different concentrations $(0,100,200,300$, and $400 \mu \mathrm{M})$ for $48 \mathrm{~h}$, and the longest root length of seedling was measured. The results indicated that the root length decreased as ACC concentration increased, and 30 out of 430 salt-tolerant plants including the lines of 
$1-29,2-7,1-105,1-119$, and 1-98 significantly exhibited ethylene insensitivity at seedling stage (Fig. 2G $-1)$.

The plant growth of the selected wheat lines 2-7, 1-119, and 1-29 in response to salt stress in soil was examined. One-week-old seedlings were planted in pots and watered with solution with different concentrations of $\mathrm{NaCl}(0,100,200,300 \mathrm{mM})$ for 30 days. The fresh weight and dry weight were measured, and the results showed that the wheat seedling growth was getting worse as the $\mathrm{NaCl}$ concentration was increased. Obviously, the fresh weight and dry weight of the salt-tolerant lines were higher than those of the wild type (Fig. 3A - C).

The MDA, Pro contents and SOD, CAT, POD activities were also compared between the salt-tolerant lines and the wild type. The results showed that salt treatment increased the MDA, Pro contents and SOD, CAT, POD activities in all samples (Fig. 3D - H). The Pro contents and SOD, CAT, POD activities in salt-tolerant lines after salt treatment were significantly higher than the wild type, and the MDA contents of the lines were significantly lower than the wild type due to salt treatment, while most of them have no difference before $\mathrm{NaCl}$ treatment (Fig. 3D - H). All these results indicated that the new wheat lines improved their salt tolerance by accumulation of Pro content, improvement of SOD, CAT and POD activities, and inhibition of MDA production in salt stress.

\section{Transcriptomic analysis of the responsive genes in the wheat mutant lines}

In order to explore the resistance mechanism of the new salt-tolerant wheat lines, the line 2-7 and 1-29 were chosen for transcriptome analysis. The 2-week-old seedlings of the lines treated with $150 \mathrm{mM} \mathrm{NaCl}$ or untreated samples were collected and used for RNA-seq analysis. Comparing the untreated and salt treated samples from the same line, the genes with fold change $\geq 2$ and False Discovery Rate (FDR) < 0.05 were defined as significant Differentially Expressed Genes (DEGs). A venn diagram of DEGs in multiple comparisons of WT-vs-WT-S (salt treated WT samples), 2-7-vs-2-7-S (salt treated 2-7 samples), and 1-29-vs-1-29-S (salt treated 1-29 samples) showed 3278 DEGs overlapped (Fig. 4A). The Gene Ontology (GO) and Kyoto Encyclopedia of Genes and Genomes (KEGG) enrichment analysis indicated that the overlapped DEGs were mostly affected and mainly focused on antenna proteins in photosynthesis, biosynthesis of secondary metabolites, cyanoamino acid metabolism, carotenoid biosynthesis, thiamine metabolism, and cutin, suberine and wax biosynthesis pathways including CHLOROPHYLL a-b BINDING PROTEINs (CABS), PEROXIDASEs (PERs/PODS), BETA-GLUCOSIDASES (BGLUS), CYTOCHROME P450 $707 \mathrm{~s}$ (CYP707s), ZEAXANTHIN EPOXIDASES (ZEPS), and HYDROXYMETHYLPYRIMIDINECS (THICS) (Fig. 4B - C; Supplementary material 2-4).

\section{Qrt-pcr Verification Of Transcriptome Results}

To confirm the results of RNA-seq, 12 DEGs were selected from the DEGs in multiple comparisons of WTvs-WT-S, 2-7-vs-2-7-S, and 1-29-vs-1-29-S, and their relative expression levels were examined by qRT-PCR (Fig. 5; Supplementary material 2-4). The sequences of the selected DEGs were listed in the 
Supplementary material 5, and the used primes were showed in the Supplementary material 6 . The relative expression level of the genes in different lines at different $\mathrm{NaCl}$ treatment periods were detected, and the results showed high consistance with the RNA-seq transcriptomic analysis (Fig. 4).

In addition, some stress-related genes including TaNHX1, TaNHX3, TaNHX7, TaNAC3, TaHAK1, TaZIP29, TaGSVI, TaSALT-1, TaSALT-2, and TaSALT-3 were also picked according to previously reports (Kawaura et al. 2008; Lu et al. 2014), and their relative expression levels in different $\mathrm{NaCl}$ treatment periods were detected by qRT-PCR in this study. The results indicated that the expressions of salt-related genes in the salt-tolerant lines were higher than the wild type (Fig. $6 \mathrm{~F}-0$ ). Together, all these results confirmed the salt tolerance of the selected wheat lines.

As the salt tolerant lines displayed decreased ethylene sensitivity, the relative expression levels of the ethylene related ETHYLENE RESPONSE FACTORs (ERFS) (TaERF1, 2, 3, 4 and 6) with different salt treatments were examined (Fig. 6A - E). The results indicated that most of the wheat lines had higher expression levels of TaERFs even without $\mathrm{NaCl}$ treatment, and the salt stress significantly increased the expression levels of the ERFs in most of the salt tolerant lines (Fig. 6A - E).

Expression characteristics and structural variation of novel wheat ERF genes in response to ethylene and salt stress

Based on the results of transcriptome sequencing, 9 novel wheat ERFs in response to ethylene and salt stress were identified in this research, and their CDS sequences and full DNA sequences were showed in the Supplementary material 7. The basic physical and chemical properties of the selected ERFs including molecular weight, theoretical pl, instability index, and aliphatic index were detected using ProtParam (http://web.ExPASy.org/ProtParam/), and the results were summarized in the Supplementary material 8. The phylogenetic map and exon-intron structure analysis showed that these 9 ERFs have diverse gene structures (Fig. 7A). However, these 9 ERFs all contained conserved GCC-box binding domain, indicating similar genes were activated by them (Fig. 7B). Heatmap analysis of the ERFs in transcriptional sequencing showed that the expression levels of 2 ERFs TRIAE_CS42_5DL_TGACv1_432926_AA1394650 and TRIAE_CS42_6AS_TGACv1_486327_AA1559760 decreased significantly after salt treatment, while the expression levels of the other 7 ERFs increased significantly after salt treatment, suggesting that they may play opposite roles in ethylene regulated salt tolerance in wheat (Fig. 7C).

\section{Discussion}

\section{Screening for the salt-tolerant wheat lines with ethylene insensitivity was efficient and effective}

Wheat (Triticum aestivum L.) is a staple crop in the world, but is only moderately salt tolerant [6]. However, salt stress was reported to affect one-fifth of irrigated agricultural land in the world [7], it is of great importance to cultivate salt-tolerant varieties in order to improve the global wheat production. In this 
research, new wheat germplasm was generated by EMS mutagenesis based on a popular wheat variety 'Luyuan 502'. The wheat variety is a semi-winter mid-maturing variety with cuboid spikes, long awns, white shells, white grains, strong growth, and excellent resistance to cold stress, rust and powdery mildew diseases, and is wildly planted in the northern part of Chinese Huanghuai winter wheat region, including Shandong Province, central and southern Hebei Province, and central and southern Shanxi Province of China. In this study, a large mutant pool was generated and screened for salt resistant lines.

Ethylene is one of plant hormones and plays an important role in plant salt-tolerance [29, 33, 34]. In dicotyledonous plants, the etiolated seedlings treated with ethylene precursor ACC showed inhibition of root and hypocotyl elongation, swelling of the hypocotyl and exaggeration of the apical hook, which is known as 'triple response' $[35,36]$. However, in monocotyledonous plants, the responses is more complex. For example, in rice, ethylene only inhibits root growth, but promotes coleoptile growth of etiolated rice seedlings, which is collectedly known as 'double response' [33, 37-39]. Unfortunately, less has been known about ethylene related responses in wheat. In the present study, we tested the mutagenized wheat lines and found their root length was sensitive to the ethylene treatment. As a result, we used the root length of etiolated wheat seedlings to screen the ethylene insensitive mutants (Fig. 2G - I). Based on the relationship between ethylene insensitivity and salt tolerance in wheat, we screened the EMS-

mutagenized pool and obtained 11 salt-tolerant wheat lines with high yield (Table 1). Our results proved the efficiency and effectiveness of the screening for salt tolerance and ethylene insensitivity in the wheat.

\section{Transcriptomic analysis for alteration of the responsive gene expressions in salt-tolerant pathway}

It was reported that salt stress induces ion imbalance and hyperosmotic effects to increase ROS concentration, damage chloroplast construct, exaggerate enzyme inefficiency, decrease photosynthesis, and accelerate photorespiration $[9,11,40]$. Plants also evolve in several responses to alleviate the negative effects of salt stress, which contribute to survival of plants in the salt stress. The salt-tolerant mechanism of new wheat mutant lines was excavated by transcriptome analysis in this research.

The transcriptomes of 2-week-old seedlings of the ethylene-insensitive salt-tolerant wheat lines 2-7 and 1-29 treated with $150 \mathrm{mM} \mathrm{NaCl}$ were compared with those of the wild type samples. The multiple comparisons of WT-vs-WT-S, 2-7-vs-2-7-S, and 1-29-vs-1-29-S showed the expression of CABs, PERS/PODs, BGLUs, CYP707s, ZEPS, and THICs in the antenna proteins in photosynthesis, biosynthesis of secondary metabolites, cyanoamino acid metabolism, carotenoid biosynthesis, thiamine metabolism, and cutin, suberine and wax biosynthesis pathways were significantly changed (Fig. 4; Supplementary material 2-4).

CABs bind chlorophyll $a$ and $b$ to make up light harvesting antenna complex, which absorbs light and transfer excitation energy to photosystems I and II in chloroplasts [41]. In high plants, CABs are encoded by multiple genes in high plants. Such as, Arabidopsis contains $10 \mathrm{CABs}$, and tomato has $16 \mathrm{CABs}$ in the genome $[41,42]$. Recently, it was reported that the expression of $C A B s$ is induced by salt, indicating a significant role of $C A B s$ in salt resistance [43]. In this research, $40 C A B s$ including TRIAE_CS42_1AL_TGACv1_000383_AA0010660, TRIAE_CS42_1AL_TGACv1_000708_AA0017440, 
TRIAE_CS42_1BL_TGACv1_030603_AA0095440, TRIAE_CS42_1BL_TGACv1_032695_AA0133030, TRIAE_CS42_1DL_TGACv1_061419_AA0194670, and TRIAE_CS42_1DL_TGACv1_061593_AA0199400 were showed in the salt-tolerant pathway of wheat (Supplementary material 2-4). Due to the limited information about $C A B s$ in salt response, more studies definitely need to be done in the future.

PERs/PODs are enzymes that catalyze substrate oxidation with hydrogen peroxide as an electron receiver. In this research, 32 PERs/PODs including TRIAE_CS42_5DL_TGACv1_434349_AA1434390, TRIAE_CS42_5DL_TGACv1_435881_AA1455860, TRIAE_CS42_5DL_TGACv1_436164_AA1458860, TRIAE_CS42_6AL_TGACv1_472947_AA1527400, and TRIAE_CS42_7AL_TGACv1_556904_AA1773120, were detected in the salt-tolerant pathway (Supplementary material 2-4).

The BGLUs belong to subfamily I of glycoside hydrolases, which hydrolyze beta-glycosidic bonds to release terminal glucosyl residues from glycosides, oligosaccharides, and disaccharides [44]. The BGLUs have been proved playing roles in plant growth, development, and biotic and abiotic resistance by releasing glucose from oligosaccharides in cell wall polysaccharides to change cell wall structures, activating defense compounds from inactive glycosides to defense against herbivores and fungi, releasing plant hormones from its inactive glyconjugates to satisfy the plants requirement, or releasing scent compounds from involatile precursor [45-47]. The specific function of a certain BGLU enzyme in plant growth, development, biotic and abiotic resistance depends on its expression pattern, substrate specificity, and different localization [48]. The BGLUs also have been reported induced by salt treatment. For example, the CsBGLU12 in Crocus sativus was significantly induced by salt, and its transient overexpression leaves of tobacco accumulated antioxidant flavonols, which confer tolerance to salt stresses by alleviation ROS accumulation [46]. In this research, 41 BGLUs including TRIAE_CS42_2BL_TGACv1_130794_AA0418050, TRIAE_CS42_3B_TGACv1_222633_AA0768330, TRIAE_CS42_3B_TGACv1_223344_AA0780610, TRIAE_CS42_3DL_TGACv1_250039_AA0861010, TRIAE_CS42_4AS_TGACv1_307764_AA1023360, and TRIAE_CS42_5AL_TGACv1_374195_AA1193240, were detected (Supplementary material 2-4). Considering the diverse functions of BGLUs in plants, the molecular mechanism of these BGLUs in salt-tolerance pathway definitely need to be explored.

WRKY transcription factors, which regulate genes by binding to a DNA cis-acting element called W-box, have important roles in response to salt stress [49]. Overexpression of the wheat TaWRKY2 and TaWRKY19 in Arabidopsis led to stronger salt responses [50]. It was also reported that salt induced expression of TaWRKY10, and overexpression of TaWRKY10 in tobacco led to enhance salt resistance including increased germination rate, root length, survival rate, relative water content, proline production, and soluble sugar contents, as well as decreased ROS and MDA contents [51]. In addition, TdWRKY1, 3, 4, and 5 isolated from durum wheat were also induced by high-salt treatment, suggesting their possible roles in salt response [52]. In this research, 12 WRKYs coding genes including

TRIAE_CS42_7DS_TGACv1_623759_AA2055930, XLOC_076596,

TRIAE_CS42_1AL_TGACv1_001348_AA0029060, TRIAE_CS42_1AL_TGACv1_002809_AA0045280, and TRIAE_CS42_1DL_TGACv1_062218_AA0210650, were also detected in the salt-tolerance pathway 
(Supplementary material 2-4), suggesting that they may play important roles in salt tolerance in wheat plants.

\section{The wheat ERFs in response to ethylene and salt stress}

ERF transcription factors regulate diverse biological processes in plant growth, development, abiotic and biotic stress responses by activating genes with GCC-box in the promoter [53, 54]. To date, 117 ERFs have been identified in bread wheat and only few ERFs including TaERF1, TaERF3, TaPIE1, TaPIEP1, TaERF4, and TaERF8-2B have been characterized. In details, TaERF8-2B, playing roles in plant growth and development, regulated plant architecture and yield related traits, and associates with plant height, heading date and 1000 kernel weight (TKW) [55]. Overexpression of TaPIE1 in wheat accumulated high soluble sugars and proline contents, and improved freezing and necrotrophic pathogen Rhizoctonia cerealis tolerance [56]. Overexpression of ERF transcription factor TaPIEP1 in wheat enhanced resistance to fungal pathogen Bipolaris sorokiniana [57].

The wheat ERF transcription factors were also reported playing roles in abiotic resistance. Overexpression of TaERF3 in bread wheat promoted tolerance to salt and drought stresses [58]. TaERF1 contributes to increase drought, cold, and salt tolerance in transgenic Arabidopsis plants [59]. While another ERF transcription factor TaERF4 might have opposite roles with TaERF1 and TaERF3 in plant abiotic tolerance, it was reported that overexpression of TaERF4 in Arabidopsis enhanced salt sensitivity, indicating the complex roles of ERF transcription factors in plant abiotic resistance [60].

Based on transcriptome sequencing, 9 novel wheat ERFs in response to ethylene and salt stress were identified in this research. Heatmap analysis showed that the expression levels of 2 ERFs TRIAE_CS42_5DL_TGACv1_432926_AA1394650 and TRIAE_CS42_6AS_TGACv1_486327_AA1559760 decreased significantly after salt treatment, suggesting they may paly negative regulation roles in ethylene regulated salt tolerance in wheat (Fig. 7C). At the same time, the expression levels of the other 7 ERFs including TRIAE_CS42_3DL_TGACv1_250510_AA0869450,

TRIAE_CS42_6DL_TGACv1_526341_AA1680010, TRIAE_CS42_7AL_TGACv1_556681_AA1768690, TRIAE_CS42_7AS_TGACv1_569387_AA1814810, TRIAE_CS42_7DL_TGACv1_606411_AA2010000, TRIAE_CS42_7DS_TGACv1_621751_AA2025220, and TRIAE_CS42_7DS_TGACv1_623962_AA2057840, increased significantly after salt treatment, indicating their positive roles in ethylene regulated salt tolerance in wheat (Fig. 7C). Comparing with the previous results, it is suggested that the wheat ERFs may function differently in regulation of stress responses. Considering most of ERF transcription factors improve abiotic tolerance in crops without causing undesirable growth phenotypes [53], it is possible to manipulate the ERFs in response to ethylene and salt stress for improvement of wheat salt-tolerance breeding in the future.

\section{Methods}

Plant material, growth condition in field, and EMS mutation 
The bread wheat (Triticum aestivum L.) cultivar 'Luyuan502' (approved by the Chinese National Crop Variety Examination and Approval Committee, 2011) used for EMS mutagenesis was bought from the market of shandong province, China. About 90,000 seeds of 'Luyuan502', designated as $\mathrm{M}_{0}$ seeds, were selected and sterilized in $2 \% \mathrm{NaClO}$ for $10 \mathrm{~min}$, washed in sterile water for $3 \mathrm{~min}$. After washed 5 times, the seeds were soaked in sterile water in the dark for 24 hours. Then, the surface sterilized seeds were incubated in $0.4 \%$ EMS solution with gentle agitation $(50 \mathrm{rpm})$ for $24 \mathrm{~h}$ at room temperature. After washed 3 times in distilled water, the seeds were sowed in the saline soil of farm in Maotuo Village, Lijin County, Dongying City, Shandong Province, China. Every single plant with excellent growth was harvested in the saline soil field with total of $0.3-0.7 \%$ salts, separately. The harvested $M_{1}$ seeds and its progeny were propagated and grown in salinized soil (Lijin, or Wudi of Shandong province) or non-saline land (Yanzhou of Shandong province) in the following years for production analysis.

\section{Ethylene, salt and drought sensitivity assays}

Detected seeds were sterilized in $2 \% \mathrm{NaClO}$ for 10 min, washed in sterile water for 3 min, repeated 5 times, and then placed in the petri dish filled with two layers of filter paper and supplemented with ethylene precursor ACC at $300 \mu \mathrm{M}$. Petri dishes were wrapped in aluminum foil and incubated at room temperature for $24 \mathrm{~h}$ or $6 \mathrm{~d}$. Ethylene insensitive mutants were screened by measuring root length of etiolated wheat seedlings. Three replicates were used, and at least 10 seeds were examined in each replicate.

Sterilized seeds were placed in the petri dish filled with two layers of filter paper and supplemented with $\mathrm{NaCl}$ at $150 \mathrm{mM}$ or mannitol at $300 \mathrm{mM}$. These petri dishes were then incubated in the growth chamber at $25^{\circ} \mathrm{C}$ under 16-h light/ 8-hour dark for $24 \mathrm{~h}$ or $6 \mathrm{~d}$. Salt or drought tolerant mutants were screened by measuring the hypocotyl and root length of wheat seedlings. Three replicates were used, and at least 10 seeds were examined in each replicate.

\section{Salt/drought treatment and physiological indicators detection}

Three salt tolerant wheat lines 2-7, 1-119, and 1-29 were selected and detected in details. The seeds of wild type 'Luyuan502' and its three salt tolerant lines 2-7, 1-119, and 1-29 were soaked in sterile water overnight and then planted in the peat-based compost supplemented with fertilizer in growth chamber at $25^{\circ} \mathrm{C}$ under 16 -h light/ 8-hour dark for $7 \mathrm{~d}$. Then the seedlings were irrigated by $0,100,200,300 \mathrm{mM} \mathrm{NaCl}$ for examination of seedling growth, dry or fresh weight.

Sterilized seeds placed in the petri dish filled with two layers of filter paper, were incubated in the growth chamber at $25^{\circ} \mathrm{C}$ under 16 -h light/ 8-hour dark for $6 \mathrm{~d}$. The seedlings were then treated with $150 \mathrm{mM} \mathrm{NaCl}$ or $300 \mathrm{mM}$ mannitol for $24 \mathrm{~h}$. The treated seedlings were used for the SOD, POD, MDA, and CAT activity detection as previously described [61-63]. Three replicates were used in the assays.

\section{qRT-PCR analysis}


Total RNA was extracted and the cDNA was synthesized as previously described [64]. The TaACTIN (GenBank: AB181991) was used as the endogenous control and the used primers were listed in Supplementary material 6 . The PCR program was performed as previously described [64]. Three technical and three biological replicates were used in the experiments.

\section{Transcriptome sequencing}

The wheat (Triticum aestivum L.) cultivar 'Luyuan502' and the salt-tolerant lines 2-7 and 1-29 was used for transcriptome analysis. The 2-week-old wheat seedlings cultivated in hydroponic solution were treated with $150 \mathrm{mM} \mathrm{NaCl}$ for 24 hours, using corresponding untreated samples as control. The salt treated and untreated seedlings were collected and sent to the Genedenovo Biotechnology Co., Ltd (Guangzhou, China) for sequencing analysis. Three replicates were used in each sample and the sequencing libraries were generated using NEBNext ${ }^{\circledR}$ Ultra ${ }^{T M}$ RNA Library Prep Kit for Illumina ${ }^{\circledR}$ (NEB, USA). The library was sequenced by Illumina HiSeq ${ }^{\mathrm{TM}} 2500$. The obtained reads were filtered by short reads alignment tool Bowtie2 2.2.8 [65], and then aligned to the reference genome by TopHat2.1.1 [66]. The reference genome was downloaded from EnsemblPlants release-32 (http://plants.ensembl.org/index.html) [67]. The transcripts were constructed, annotated and identified by Reference Annotation Based Transcript (RABT) program, Cufflinks2.2.1, and TopHat2.1.1 [68, 69].

The genes with fold change $\geq 2$ and FDR $<0.05$ were defined as significant DEGs. Their GO functions and KEGG pathways were further detected according to the GO database (http://www.geneontology.org/) and KEGG database (http://www.genome.jp/kegg/), respectively. The genes of transcription factors were identified using software iTAK1.2 [70].

\section{Characterization of ERFs in response to ethylene and salt stress}

The ERFs in response to ethylene and salt stress were identified and selected according to the results of transcriptome sequencing, and the basic physical and chemical properties of the selected ERFs including molecular weight, theoretical pl, instability index, and aliphatic index were analyzed using ProtParam (http://web.ExPASy.org/ProtParam/). Multiple sequence alignments of selected ERFs were created using Clustal $\mathrm{X}$, and then used as the input for the neighbor-joining algorithm in MEGA 7.0 to construct phylogenetic tree [71]. GSDS (http://gsds.cbi.pku.edu.cn/) was used to analyze the exon-intron structure of selected ERFs, and SMART (http://smart.embl-heidelberg.de/) was used to identify the GCC-box binding domain in ERFs [72]. Heatmap of the expression level of selected ERFs in transcriptional sequence was constructed by website software in omicshare (https://www.omicshare.com/tools/). The red color indicated high-expressed genes, and blue indicated low-expressed genes.

\section{Statistical analyses}

Statistical analyses were performed by SAS, and the statistical significance of the difference was evaluated by ANOVA. 


\section{Abbreviations}

ABA

Abscisic Acid; ACC:Aminocyclopropane-1-Carboxylate; AUX:Auxin; BGLU:Beta-Glucosidase; BR:Brassinosteroid; CAB:Chlorophyll a-b Binding Protein; CAT:Catalase; CK:Cytokinin; CTR:Constitutive Triple Response; CYP:Cytochrome P450; DEG:Differentially Expressed Gene; EBF:EIN3 binding F-box; EIL:EIN3-Like; EIN:Ethylene Insensitive; EMS:Ethylmethylsulfonate; ERF:Ethylene Response Factor; ET:Ethylene; ETO:Ethylene Overproducer; ETR:Ethylene Response; FDR:False Discovery Rate; GA:Gibberellin; GO:Gene Ontology; JA:Jasmonate; KEGG:Kyoto Encyclopedia of Genes and Genomes; MDA:Malonic Dialdehyde; NCED:9-cis-epoxycaroteniod dioxygenase; PER:Peroxidase; POD:Peroxidase; RABT:Reference Annotation Based Transcript; ROS:Reactive Oxygen Species; SA:Salicylic Acid; SL:Strigolactone; SOD:Superoxide Dismutase; THI:Hydroxymethylpyrimidine; ZEP:Zeaxanthin Epoxidase

\section{Declarations}

\section{Acknowledgments}

We greatly appreciate the contributions of Wenqiang Pan, Yang Xue, Jiacai Chen, Jingli Yang, Jinnan Song, Yaqi Wang, Xuehua Liu, Zhongxin Wang, Longfei Qiao, Yuhang Zhao, and the others for the experiments of seed mutagenesis and plant growth.

\section{Author Contributions}

QM worked on transcriptome analysis, statistical analysis, and writing of the manuscript. $\mathrm{HZ}$ contributed on the EMS mutagenesis, material collection and physiological experiments. XS and CS worked on the qRT-PCR detection. YY worked on wheat breeding and mutant screening. CHD participated in the experiments design and manuscript revision. All authors have read and approved the manuscript.

\section{Funding}

This work was supported by the National Natural Science Foundation of China (Grant No. 31870255) for transcriptomic sequencing, Shandong Natural Science Foundation (ZR2019MC061) for EMS mutagenesis and Shandong Agricultural Variety Project (2019LZGC015) for mutant screening to CHD, and the National Natural Science Foundation of China (Grant No. 31900247) to QM for qRT-PCR analysis.

\section{Availability of data and materials}

The FASTQ files of raw data were uploaded to the NCBI Sequence Read Archive (SRA), and the SRA study accession is PRJNA549107.

\section{Ethics approval and consent to participate}

Not applicable. 


\section{Consent for publication}

Not applicable.

\section{Declarations}

We declare that the authors of this paper have no conflict of interest for publication.

\section{References}

1. Dubcovsky J, Dvorak J. Genome plasticity a key factor in the success of polyploid wheat under domestication. Science. 2007;316:1862-6.

2. Huang S, Sirikhachornkit A, Su X, et al. Genes encoding plastid acetyl-CoA carboxylase and 3phosphoglycerate kinase of the Triticum/Aegilops complex and the evolutionary history of polyploid wheat. Proc Natl Acad Sci USA. 2002;99:8133-8.

3. Feldman M, Levy AA, Fahima T, et al. Genomic asymmetry in allopolyploid plants: wheat as a model. J Exp Bot. 2012;63:5045-59.

4. Breiman A,BG. Wheat evolution. Isr J Plant Sci. 1995;43:85-98.

5. Gill BS, Appels R, Botha-Oberholster AM, et al. A workshop report on wheat genome sequencing: international genome research on wheat consortium. Genetics. 2004;168:1087-96.

6. Munns R, Tester M. Mechanisms of salinity tolerance. Annu Rev Plant Biol. 2008;59:651-81.

7. Yamaguchi T, Blumwald E. Developing salt-tolerant crop plants: challenges and opportunities. Trends Plant Sci. 2005;10:615-20.

8. Hasegawa PM, Bressan RA, Zhu JK, et al. Plant cellular and molecular responses to high salinity. Annu Rev Plant Physiol Plant Mol Biol. 2000;51:463-99.

9. Liu D, Han C, Deng X, et al. Integrated physiological and proteomic analysis of embryo and endosperm reveals central salt stress response proteins during seed germination of winter wheat cultivar Zhengmai 366. BMC Plant Biol. 2019;19:29.

10. Munns R. Comparative physiology of salt and water stress. Plant Cell Environ. 2002;25:239-50.

11. Wang WQ, Liu SJ, Song SQ, et al. Proteomics of seed development, desiccation tolerance, germination and vigor. lant Physiol Biochem. 2015;86:1-15.

12. Abogadallah GM. Antioxidative defense under salt stress. Plant Signal Behav. 2010;5:369-74.

13. Szczerba MW, Britto DT, Kronzucker HJ. $\mathrm{K}^{+}$transport in plants: physiology and molecular biology. $\mathrm{J}$ Plant Physiol. 2009;166:447-66.

14. Loescher W, Chan ZL, Grumet R. Options for developing salt-tolerant crops Hortscience. 2011;46:1085-92.

15. Bari R, Jones JD. Role of plant hormones in plant defence responses. Plant Mol Biol. 2009;69:47388. 
16. Fujita M, Fujita Y, Noutoshi Y, et al. Crosstalk between abiotic and biotic stress responses: a current view from the points of convergence in the stress signaling networks. Curr Opin Plant Biol. 2006;9:436-42.

17. Horvath E, Szalai G, Janda T. Induction of abiotic stress tolerance by salicylic acid signaling. J Plant Growth Regul. 2007;26:290-300.

18. Ku YS, Sintaha M, Cheung MY, et al. Plant hormone signaling crosstalks between biotic and abiotic stress responses. Int J Mol Sci. 2018;19:3206.

19. Chen CW, Yang YW, Lur HS, et al. A novel function of abscisic acid in the regulation of rice (Oryza sativa L.) root growth and development. Plant Cell Physiol. 2006;47:1-13.

20. Park HY, Seok HY, Park BK, et al. Overexpression of Arabidopsis ZEP enhances tolerance to osmotic stress. Biochem Biophys Res Commun. 2008;375:80-5.

21. Lu Y, Li Y, Zhang J, et al. Overexpression of Arabidopsis molybdenum cofactor sulfurase gene confers drought tolerance in maize (Zea mays L.). PLoS One. 2013;8:e52126.

22. Zhang $Y$, Tan J, Guo Z, et al. Increased abscisic acid levels in transgenic tobacco over-expressing 9 cis-epoxycarotenoid dioxygenase influence $\mathrm{H}_{2} \mathrm{O}_{2}$ and $\mathrm{NO}$ production and antioxidant defences. Plant Cell Environ. 2009;32:509-19.

23. Zhang J, Yu H, Zhang Y, et al. Increased abscisic acid levels in transgenic maize overexpressing AtLOS5 mediated root ion fluxes and leaf water status under salt stress. J Exp Bot. 2016;67:133955.

24. Morgan PW, Drew MC. Ethylene and plant responses to stress. Physiol Plant. 1997;100:620-30.

25. van Loon LC, Geraats BP, Linthorst HJ. Ethylene as a modulator of disease resistance in plants. Trends Plant Sci. 2006;11:184-91.

26. Jiang C, Belfield EJ, Cao Y, et al. An Arabidopsis soil-salinity-tolerance mutation confers ethylenemediated enhancement of sodium/potassium homeostasis. Plant Cell. 2013;25:3535-52.

27. Peng J, Li Z, Wen X, et al. Salt induced stabilization of EIN3/EIL1 confers salinity tolerance by deterring ROS accumulation in Arabidopsis. PLoS Genet. 2014;10:e1004664.

28. Achard P, Cheng H, De Grauwe L, et al. Integration of plant responses to environmentally activated phytohormonal signals. Science. 2006;311:91-4.

29. Cao WH, Liu J, He XJ, et al. Modulation of ethylene responses affects plant salt-stress responses. Plant Physiol. 2007;143:707-19.

30. Cao YR, Chen SY, Zhang JS. Ethylene signaling regulates salt stress response: An overview. Plant Signal Behav. 2008;3:761-3.

31. Lei G, Shen M, Li ZG, et al. EIN2 regulates salt stress response and interacts with a MA3 domaincontaining protein ECIP1 in Arabidopsis. Plant Cell Environ. 2011;34:1678-92.

32. Wang Y, Wang T, Li K, et al. Genetic analysis of involvement of ETR1 in plant response to salt and osmotic stress. Plant Growth Regul. 2008;54:261-9. 
33. Yang C, Ma B, He SJ, et al. MAOHUZI6/ETHYLENE INSENSITIVE3-LIKE1 and ETHYLENE INSENSITIVE3-LIKE2 regulate ethylene response of roots and coleoptiles and negatively affect salt tolerance in rice. Plant Physiol. 2015;169:148-65.

34. Bahieldin A, Atef A, Edris S, et al. Ethylene responsive transcription factor ERF109 retards PCD and improves salt tolerance in plant. BMC Plant Biol. 2016;16:216.

35. Bleecker AB, Estelle MA, Somerville C, et al. Insensitivity to ethylene conferred by a dominant mutation in Arabidopsis thaliana. Science. 1988;241:1086-9.

36. Guzman P, Ecker JR. Exploiting the triple response of Arabidopsis to identify ethylene-related mutants. Plant Cell. 1990;2:513-23.

37. Ma B, Chen SY, Zhang JS. Ethylene signaling in rice. Chin Sci Bull. 2010;55:2204-10.

38. Ma B, He SJ, Duan KX, et al. Identification of rice ethylene-response mutants and characterization of MHZ7/OsEIN2 in distinct ethylene response and yield trait regulation. Mol Plant. 2013;6:1830-48.

39. Ma B, Yin CC, He SJ, et al. Ethylene-induced inhibition of root growth requires abscisic acid function in rice (Oryza sativa L.) seedlings. PLoS Genet. 2014;10:e1004701.

40. Mallik S, Nayak M, Sahu BB, et al. Response of antioxidant enzymes to high $\mathrm{NaCl}$ concentration in different salt-tolerant plants. Biol Plant. 2011;55:191-5.

41. Jansson S. A guide to the identification of the Lhc genes and their relatives in Arabidopsis. Trends Plant Sci. 1999;4:236-40.

42. Schwartz E, Stasys R, Aebersold R, et al. Sequence of a tomato gene encoding a third type of LHCII chlorophyll a/b-binding polypeptide. Plant Mol Biol. 1991;17:923-5.

43. Silva J, Kim YJ, Sukweenadhi J, et al. Molecular characterization of 5-chlorophyll a/b-binding protein genes from Panax ginseng Meyer and their expression analysis during abiotic stresses.

Photosynthetica. 2016;54:446-58.

44. Ketudat Cairns JR. Esen A. $\beta$-Glucosidases. Cell Mol Life Sci. 2010;67:3389-405.

45. Halkier BA, Gershenzon J. Biology and biochemistry of glucosinolates. Annu Rev Plant Biol. 2006;57:303-33.

46. Baba SA, Vishwakarma RA, Ashraf N. Functional characterization of CsBGlu12, a beta-glucosidase from Crocus sativus, provides insights into its role in abiotic stress through accumulation of antioxidant flavonols. J Biol Chem. 2017;292:4700-13.

47. Dharmawardhana DP, Ellis BE, Carlson JE. A beta-glucosidase from lodgepole pine xylem specific for the lignin precursor coniferin. Plant Physiol. 1995;107:331-9.

48. Rouyi C, Baiya S, Lee SK, et al. Recombinant expression and characterization of the cytoplasmic rice ß-glucosidase Os1BGlu4. PLoS ONE. 2014;9:e96712.

49. Eulgem T, Rushton PJ, Robatzek S, et al. The WRKY superfamily of plant transcription factors. Trends Plant Sci. 2000;5:199-206.

50. Niu CF, Wei W, Zhou QY, et al. Wheat WRKY genes TaWRKY2 and TaWRKY19 regulate abiotic stress tolerance in transgenic Arabidopsis plants. Plant Cell Environ. 2012;35:1156-70. 
51. Wang C, Deng P, Chen L, et al. A wheat WRKY transcription factor TaWRKY10 confers tolerance to multiple abiotic stresses in transgenic tobacco. PLoS One. 2013;8:e65120.

52. Yousfi F, Makhloufi E, Marande W, et al. Comparative analysis of WRKY genes potentially involved in salt stress responses in Triticum turgidum L. ssp. durum. Front Plant Sci. 2016;7:2034.

53. Xu ZS, Chen M, Li LC, et al. Functions and application of the AP2/ERF transcription factor family in crop improvement. J Integr Plant Biol. 2011;53:570-85.

54. Gutterson N, Reuber TL. Regulation of disease resistance pathways by AP2/ERF transcription factors. Curr Opin Plant Biol. 2004;7:465-71.

55. Zhang L, Liu P, Wu J, et al. Identification of a novel ERF gene, TaERF8, associated with plant height and yield in wheat. BMC Plant Biol. 2020;20:263.

56. Zhu X, Qi L, Liu X, et al. The wheat ethylene response factor transcription factor PATHOGEN-INDUCED ERF1 mediates host responses to both the necrotrophic pathogen Rhizoctonia cerealis and freezing stresses. Plant Physiol. 2014;164:1499-514.

57. Dong N, Liu X, Lu Y, et al. Overexpression of TaPIEP1, a pathogen-induced ERF gene of wheat, confers host-enhanced resistance to fungal pathogen Bipolaris sorokiniana. Funct Integr Genomics. 2010;10:215-26.

58. Rong W, Qi L, Wang A, et al. he ERF transcription factor TaERF3 promotes tolerance to salt and drought stresses in wheat. Plant Biotechnol J. 2014;12:468-79.

59. Xu ZS, Xia LQ, Chen M, et al. Isolation and molecular characterization of the Triticum aestivum L. ethylene-responsive factor 1 ( TaERF1) that increases multiple stress tolerance. Plant Mol Biol. 2007;65:719-32.

60. Dong W, Ai X, Xu F, et al. Isolation and characterization of a bread wheat salinity responsive ERF transcription factor. Gene. 2012;511:38-45.

61. He B, Xu H, Chen J. Effects of drought stress on the permeability of plasma membrane and antioxidation enzymes of the leaves of sweet potato. J Guangxi Agric Univ. 1997;16:287-90.

62. Zhang JS, Wang YQ, Song JN, et al. Effect of aspartic acid on physiological characteristics and gene expression of salt exclusion in Tartary buckwheat under salt stress. J Plant Biochem Biotechnol. 2019;29:94-101.

63. Zou Q. Experimental guide to plant physiology. Beijing: China Agricultural Press; 2000.

64. Ma Q, Zhang G, Hou L, et al. Vitis vinifera VvWRKY13 is an ethylene biosynthesis-related transcription factor. Plant Cell Rep. 2015;34:1593-603.

65. Langmead B, Salzberg SL. Fast gapped-read alignment with Bowtie2. Nat Methods. 2012;9:357-9.

66. Kim D, Pertea G, Trapnell C, et al. TopHat2: accurate alignment of transcriptomes in the presence of insertions, deletions and gene fusion. Genome Biol. 2013;14:R36.

67. Bolser DM, Kerhornou A, Walts B, et al. Triticeae resources in ensembl plants. Plant Cell Physiol. 2015;56:e3. 
68. Trapnell C, Roberts A, Goff L, et al. Differential gene and transcript expression analysis of RNA-seq experiments with TopHat and Cufflinks. Nat Protoc. 2012;7:562-78.

69. Trapnell C, Williams BA, Pertea G, et al. Transcript assembly and quantification by RNA-seq reveals unannotated transcripts and isoform switching during cell differentiation. Nat Biotechnol. 2010;28:511-5.

70. Zheng Y, Jiao C, Sun H, et al. iTAK: a program for genome-wide prediction and classification of plant transcription factors, transcriptional regulators, and protein kinases. Mol Plant. 2016;9:1667-70.

71. Tamura K, Peterson D, Peterson N, et al. MEGA5: Molecular evolutionary genetics analysis using maximum likelihood, evolutionary distance, and maximum parsimony methods. Mol Biol Evol. 2011;28:2731-9.

72. Zou Z, Wang R, Wang R, et al. Genome-wide identification, phylogenetic analysis, and expression profiling of the BBX family genes in pear. J Hortic Sci Biotech. 2018;93:37-50.

\section{Figures}



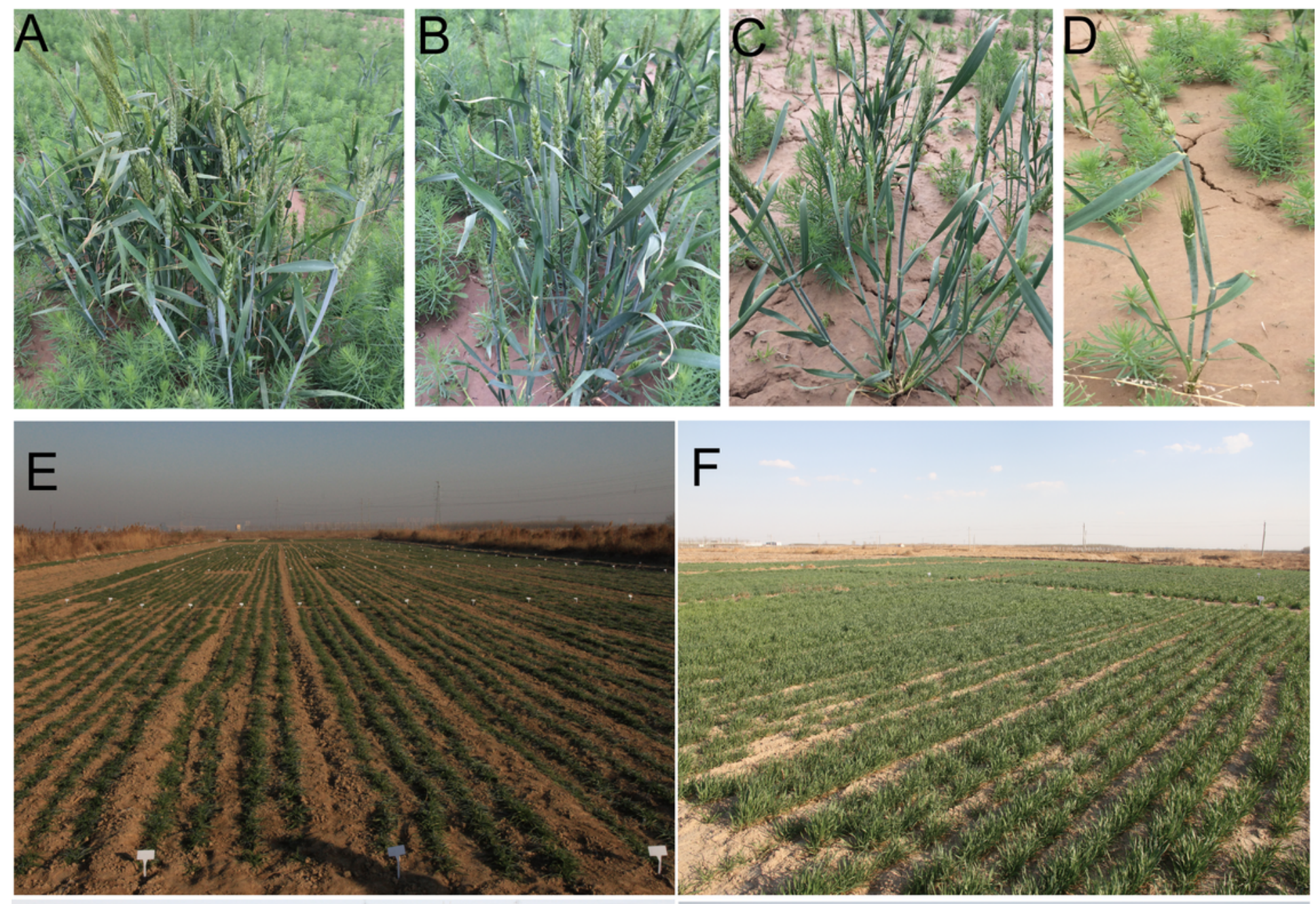

\section{G}
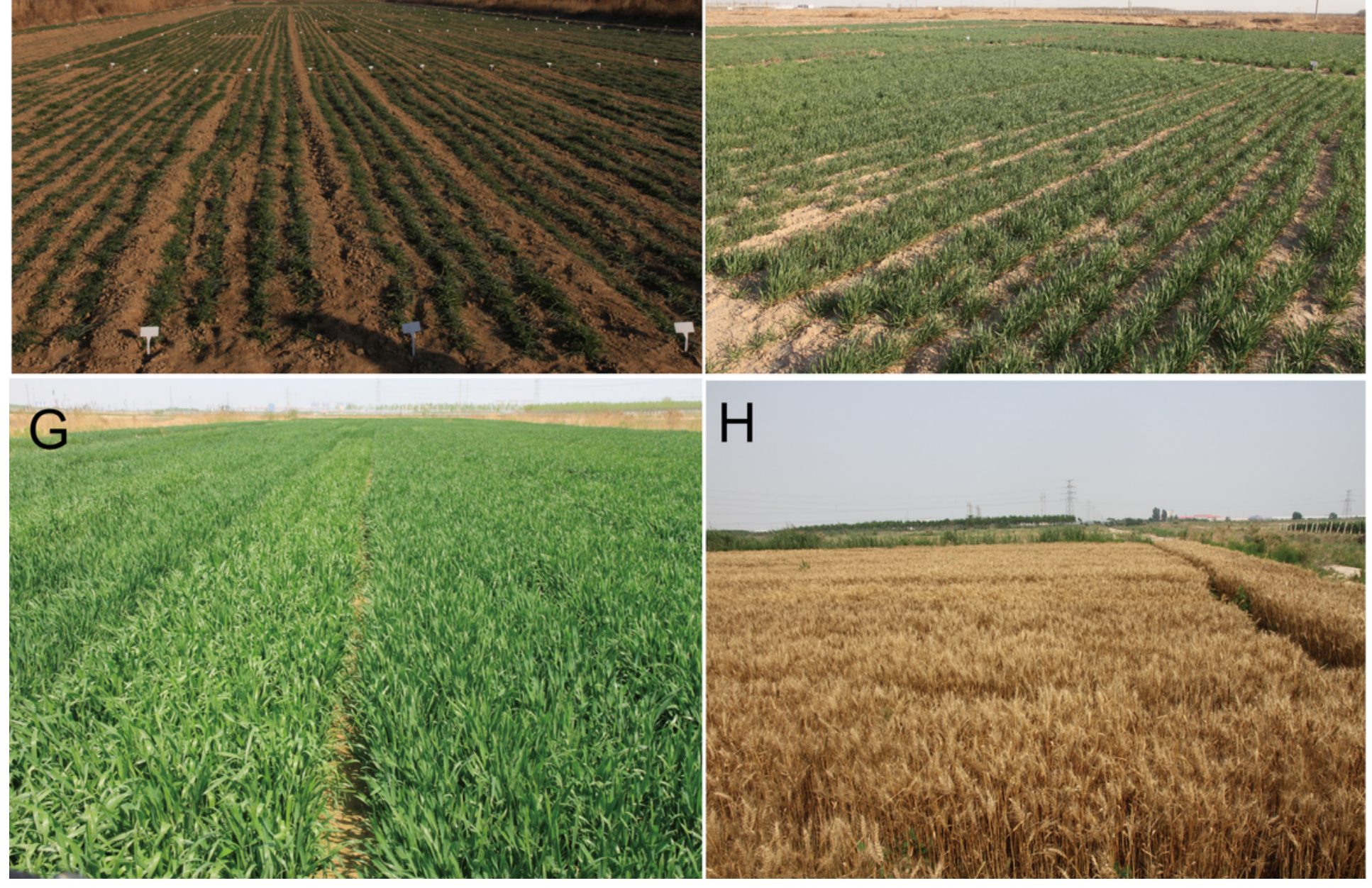

\section{Figure 1}

Growth of the EMS-mutagenized wheat lines in salinized soil. (A - D) Different EMS-mutagenized wheat plants grown in the salinized soil field of Maotuo Village, Lijin of Shandong Province in May of 2017. (E H) Propagated salt-tolerant wheat lines in the same salinized field of Maotuo on Oct 20th, 2017; Dec 1st, 2017; April 4th, 2018; and June 20th, 2018, respectively. 

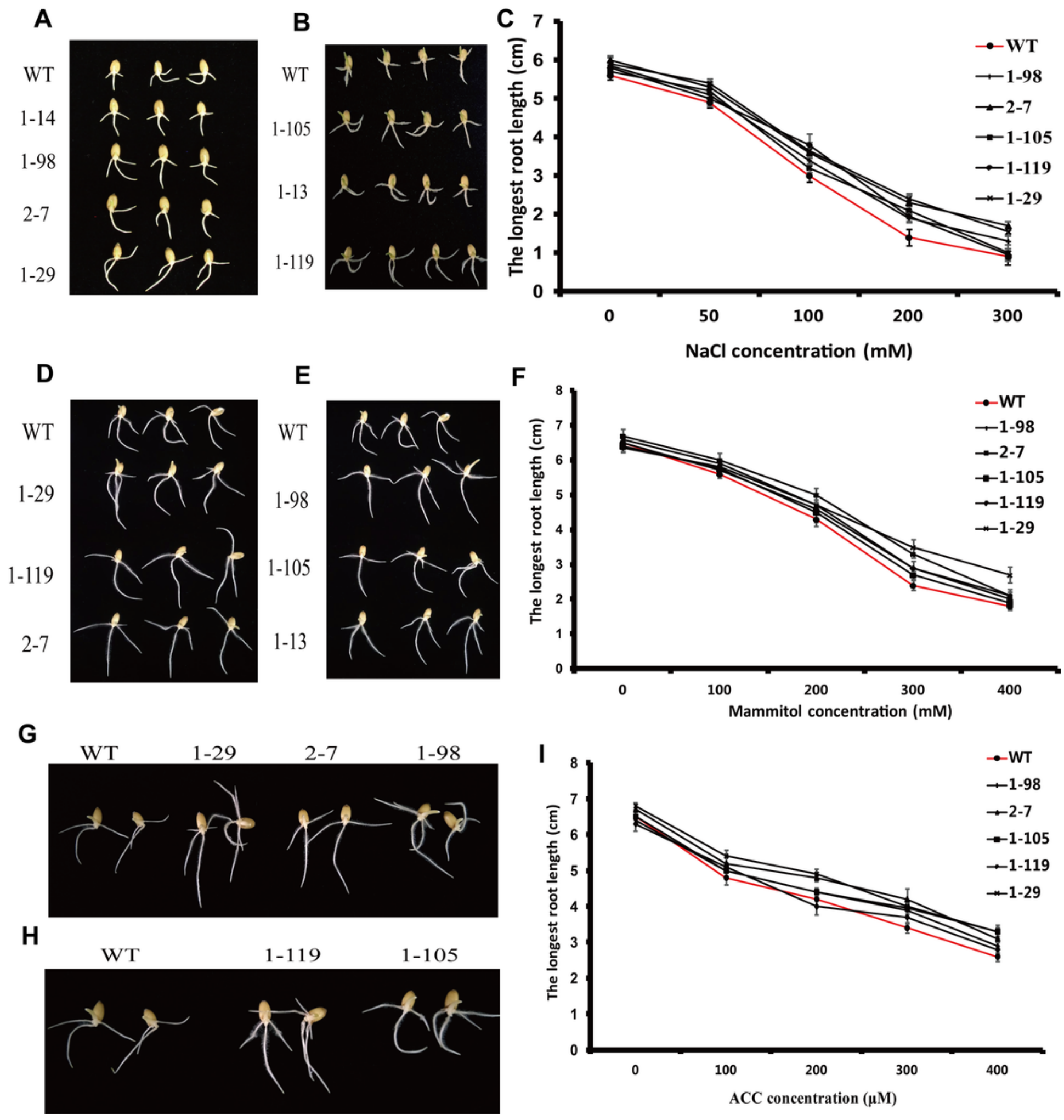

Figure 2

Stress resistance and ethylene sensitivity assays of wheat seedlings. (A - B) Salt-tolerant wheat seedlings germinated and grown in $200 \mathrm{mM} \mathrm{NaCl}$ for $48 \mathrm{~h}$. (C) The longest primary root length of salt-tolerant wheat seedlings germinated and grown in the presence of $\mathrm{NaCl}$ for $48 \mathrm{~h}$. (D - E) Wheat seedlings germinated and grown in $300 \mathrm{mM}$ mannitol for $48 \mathrm{~h}$. (F) The longest root length of salt-tolerant wheat seedlings treated with different concentrations of mannitol for $48 \mathrm{~h}$. (G - H) Etiolated wheat seedlings 
treated with $300 \mu \mathrm{M}$ ACC for $48 \mathrm{~h}$ in darkness. (I) The longest root length of wheat seedlings treated with different concentrations of ACC for $48 \mathrm{~h}$ in darkness.

A

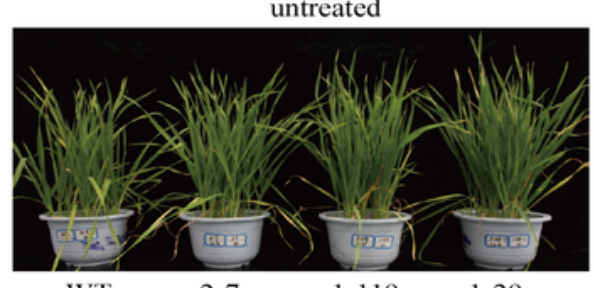

WT

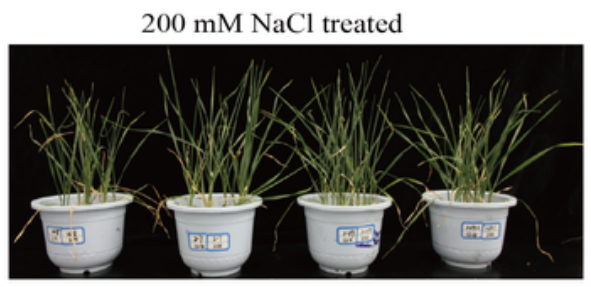

WT

$2-7$

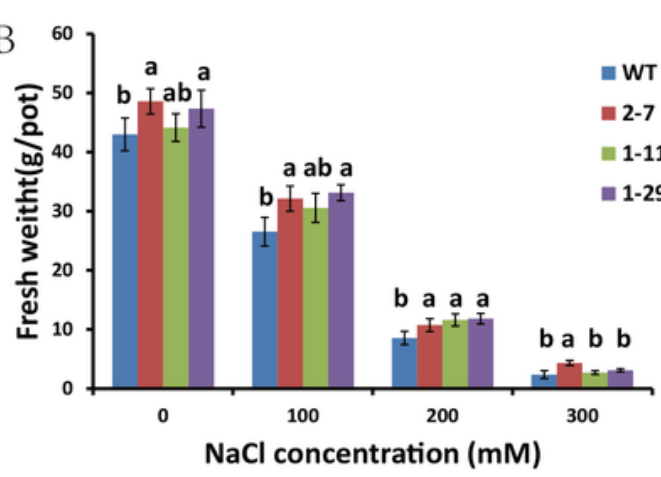

D

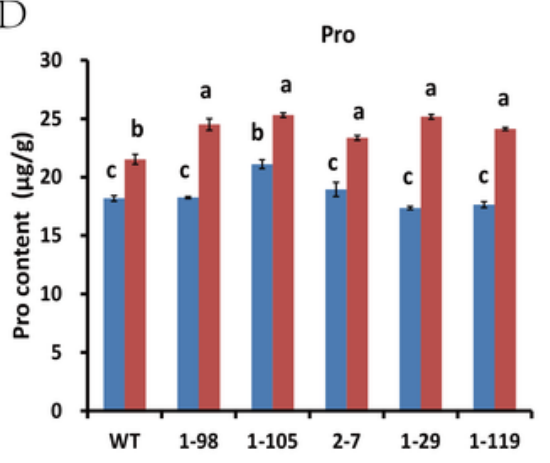

$100 \mathrm{mM} \mathrm{NaCl}$ treated

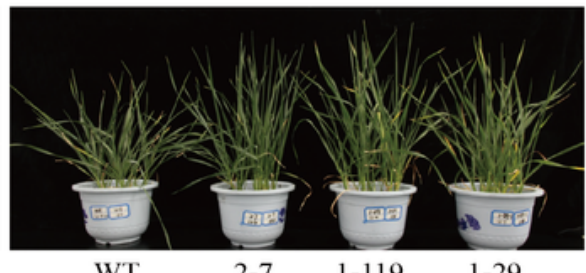

WT

$300 \mathrm{mM} \mathrm{NaCl}$ treated

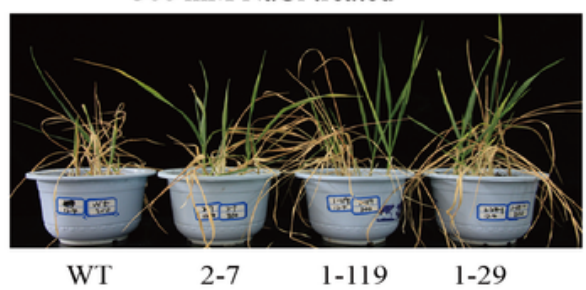

C

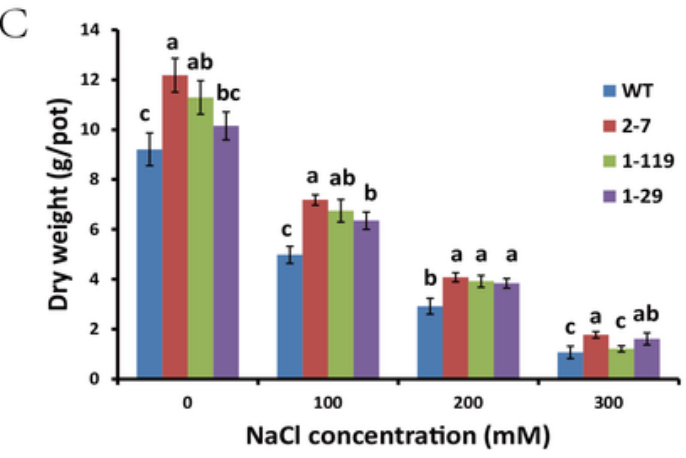

E

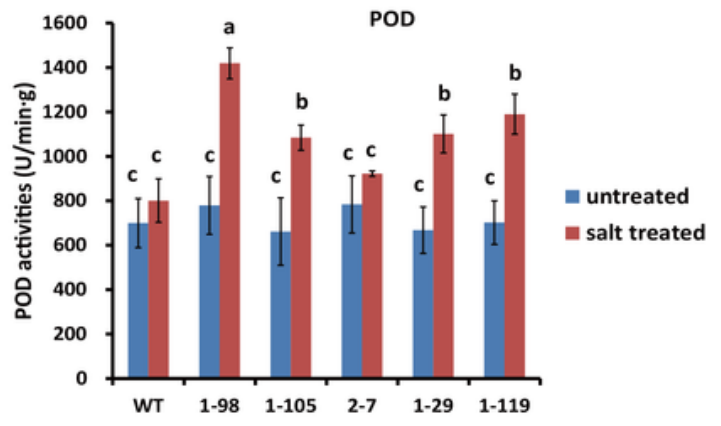

$\mathrm{H}$
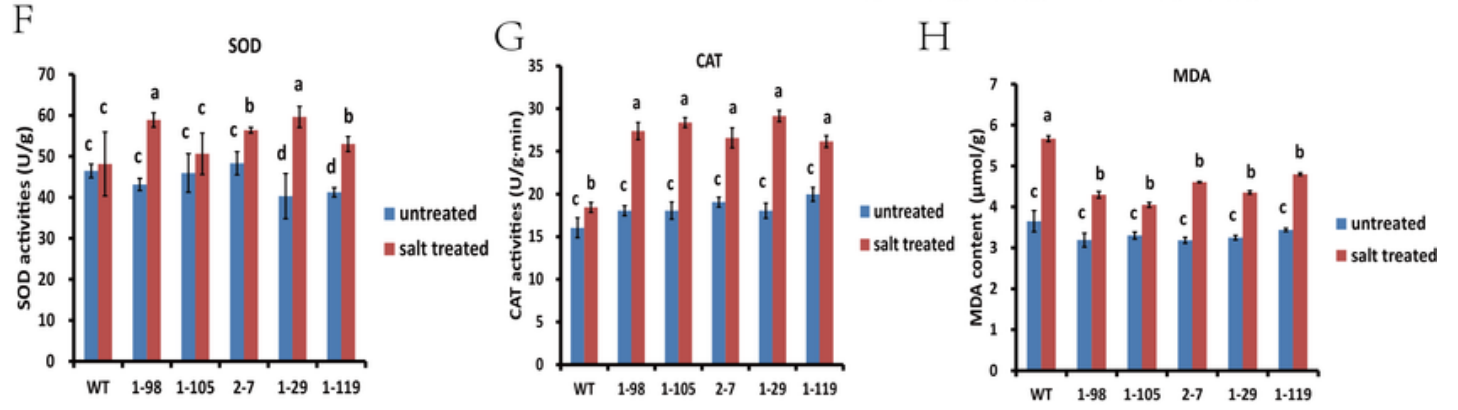

Figure 3

Salt resistance and physiological assays of the wheat lines. (A) Growth of the salt-tolerant wheat lines treated with $0,100,200$, and $300 \mathrm{mM} \mathrm{NaCl}$ for $30 \mathrm{~d}$. (B - C) Fresh weight (B) and dry weight (C) 
comparisons of the wheat lines treated with different concentrations of $\mathrm{NaCl}$. (D - H) Examination of Pro (D), POD (E), SOD (F), CAT (G), and MDA (H), in the wheat lines after $150 \mathrm{mM} \mathrm{NaCl}$ treatment for $48 \mathrm{~h}$.

A
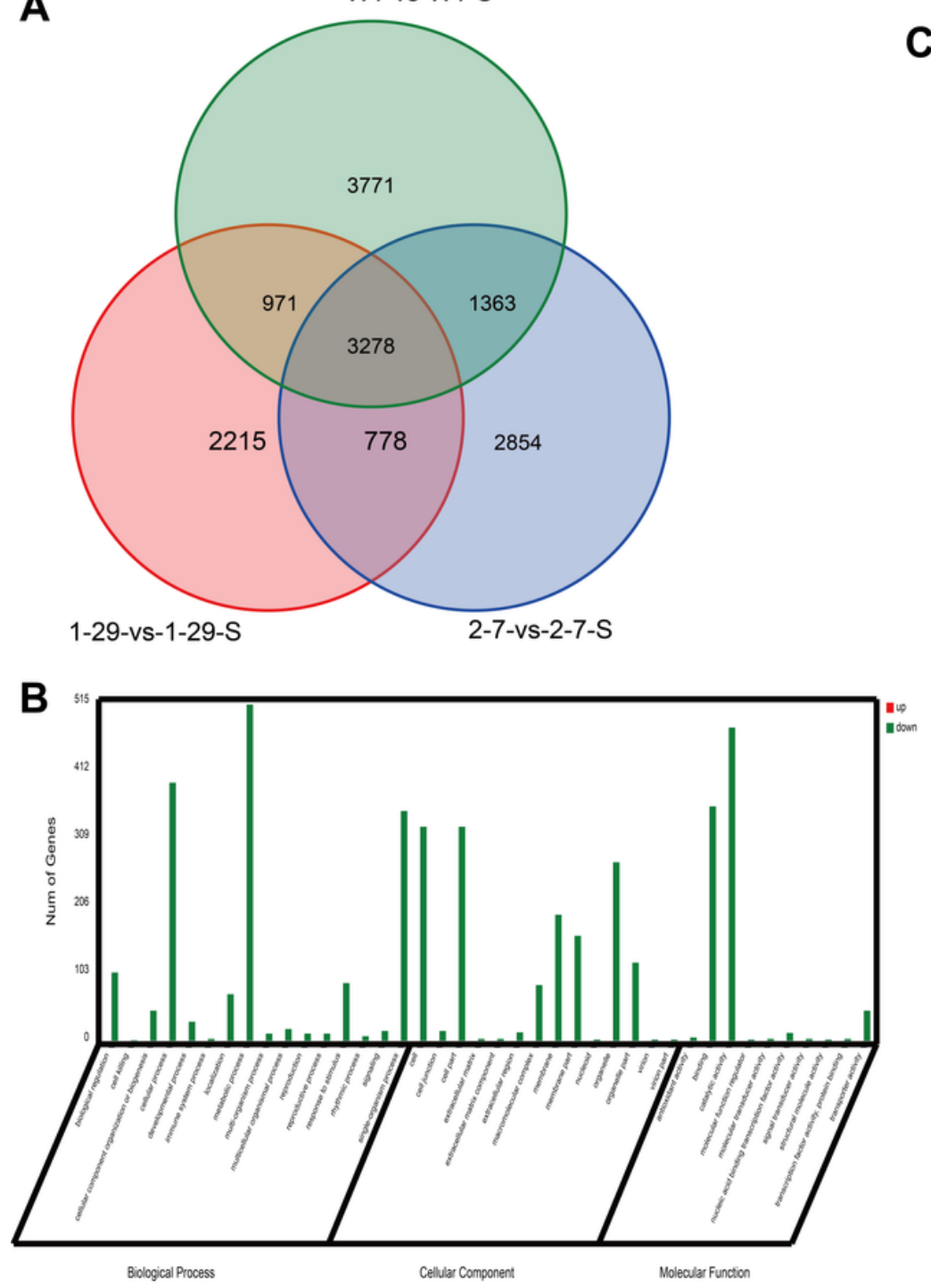

C

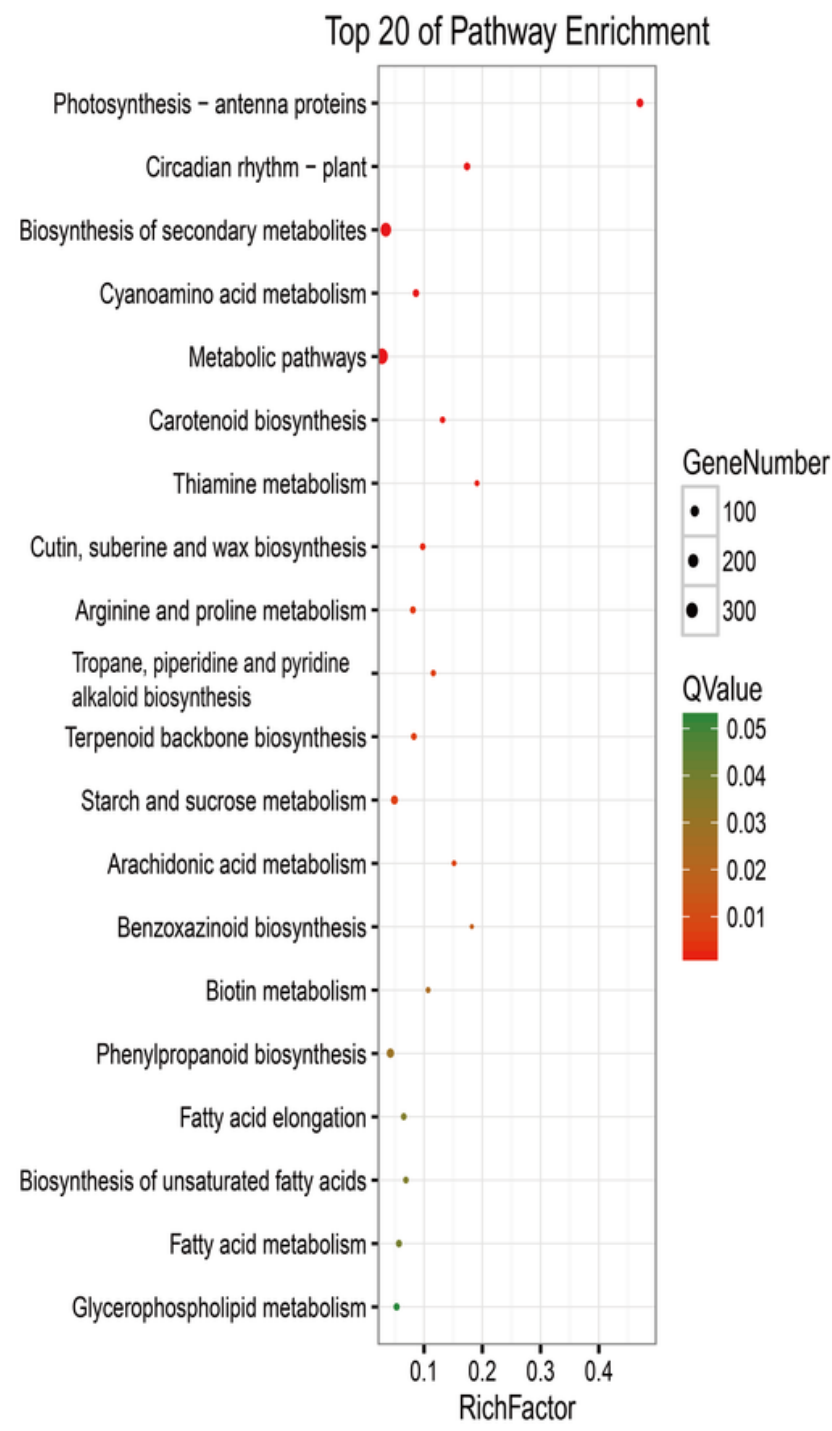

\section{Figure 4}

The multiple comparisons of WT-vs-WT-S, 1-29-vs-1-29-S, and 2-7-vs-2-7-S. (A) The venn diagrams of DEGs in the multiple comparisons. (B) The GO enrichment analysis of DEGs in the multiple comparisons. (C) The top 20 KEGG enrichment analysis of DEGs in the multiple comparisons. Rich factor is the ratio of DEG number to the total gene number, which is ranged from 0 to 1 . The bigger the Rich factor, the more significant the enrichment is. The QValue is the corrected p-value through multiple hypothesis testing. The smaller the QValue, the more significant the enrichment is. 

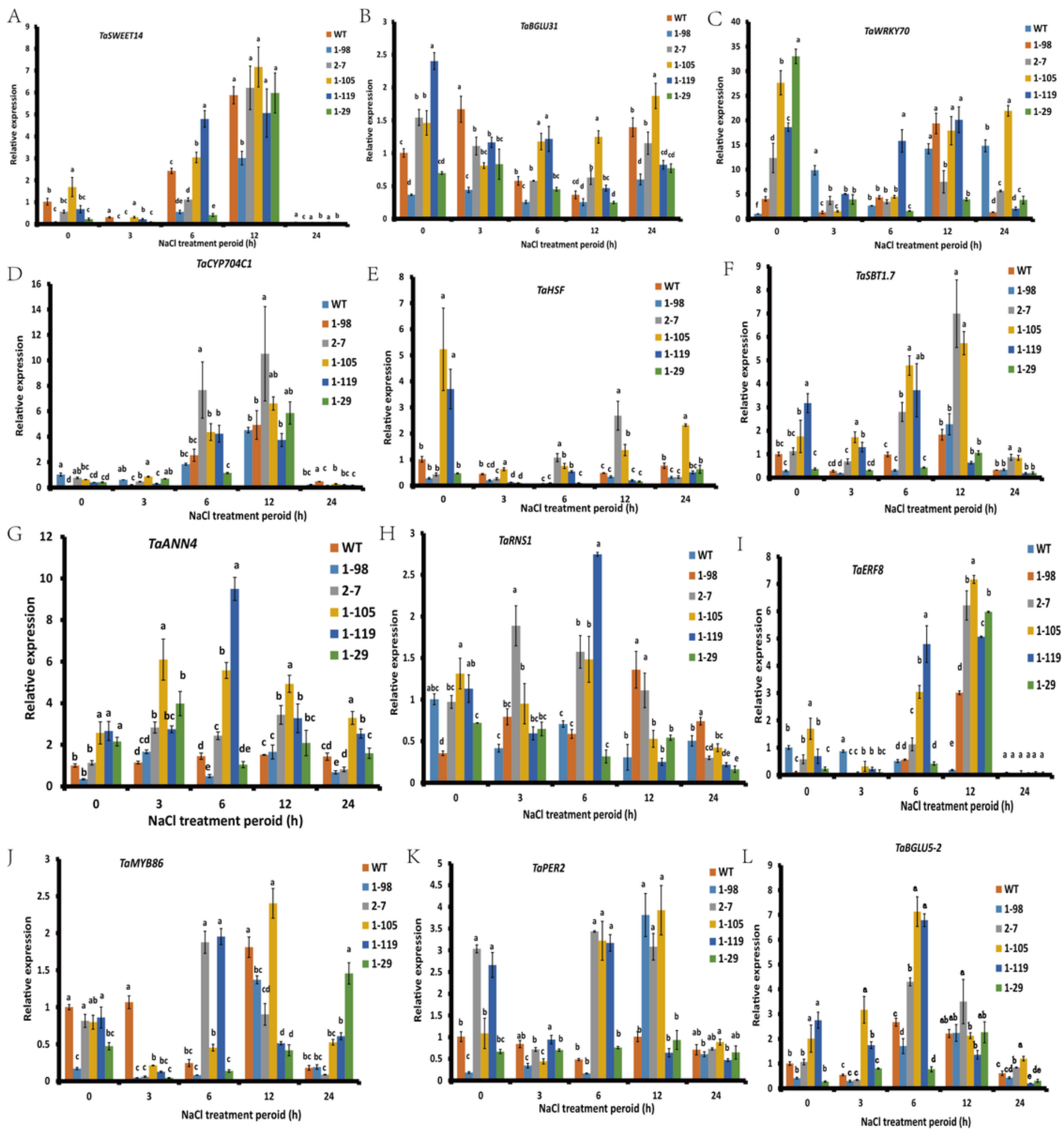

Figure 5

Expression profiles of the 12 salt-tolerant related DEGs in the wheat lines. (A - L) qRT-PCR assays for the relative expression of the 12 salt-tolerant related DEGs in different wheat plants treated with $150 \mathrm{mM}$ $\mathrm{NaCl}$ for different periods $(0-24 \mathrm{~h})$. The statistical significance of the difference was confirmed by ANOVA at $a=0.05$ level. 
A

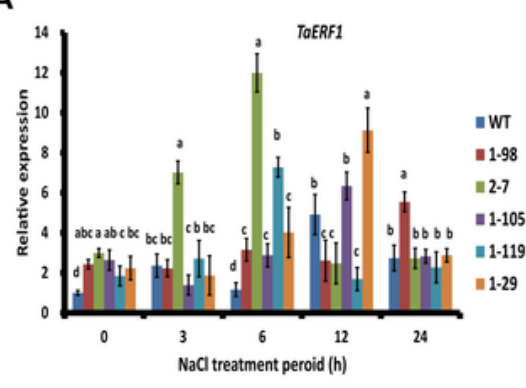

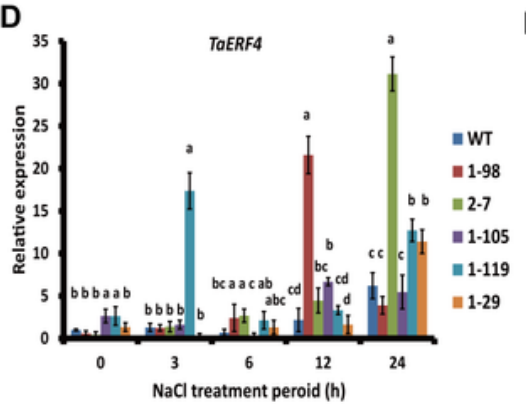
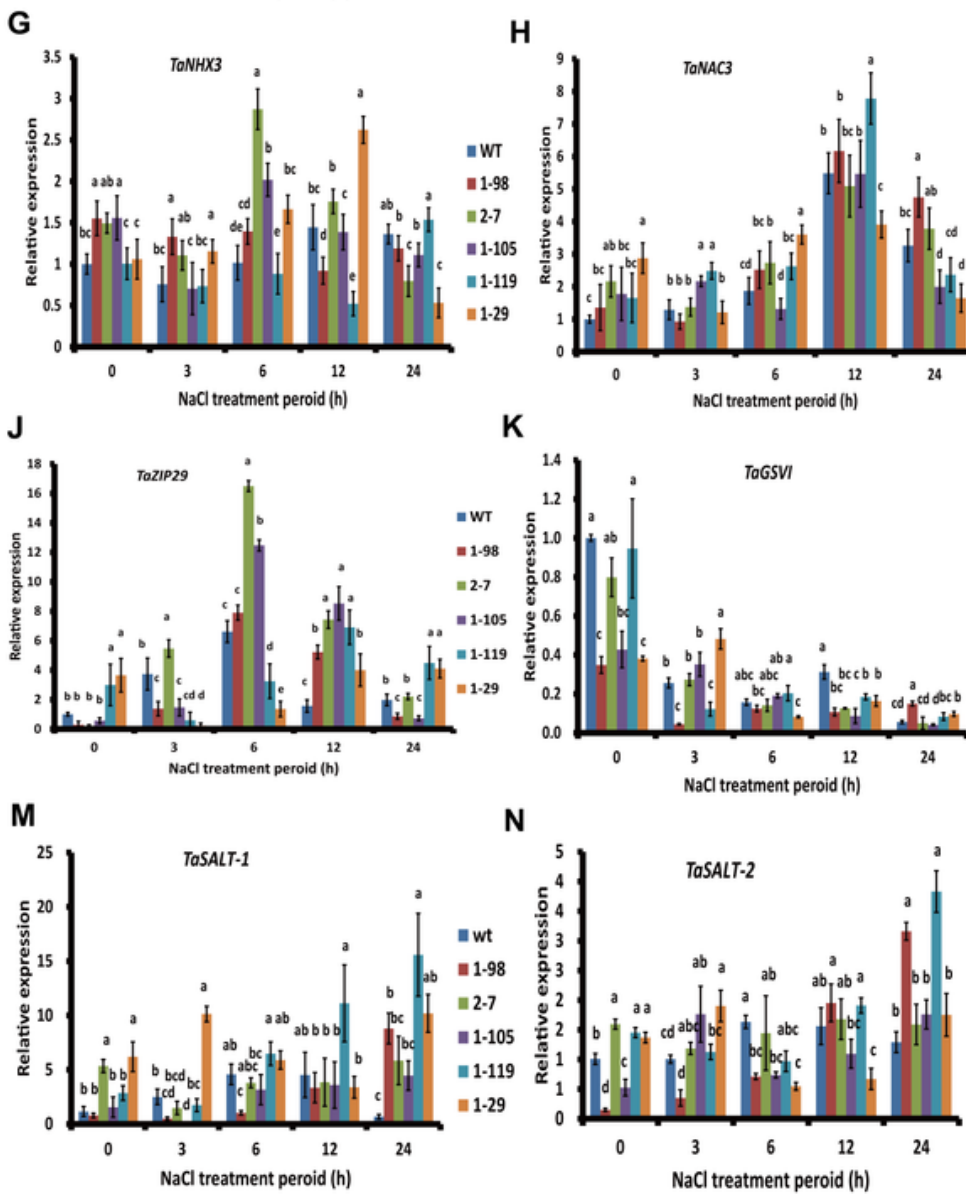

B

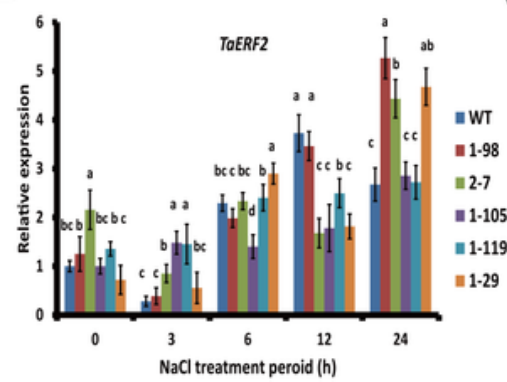

E

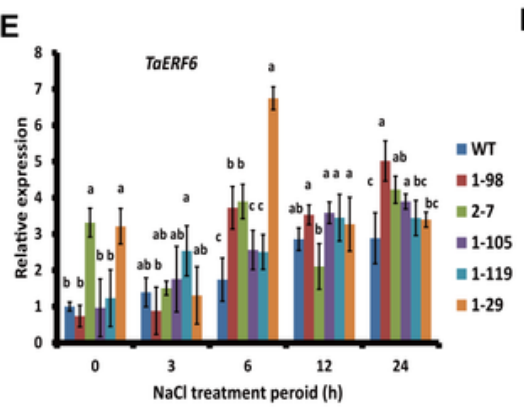

$\mathbf{F}$

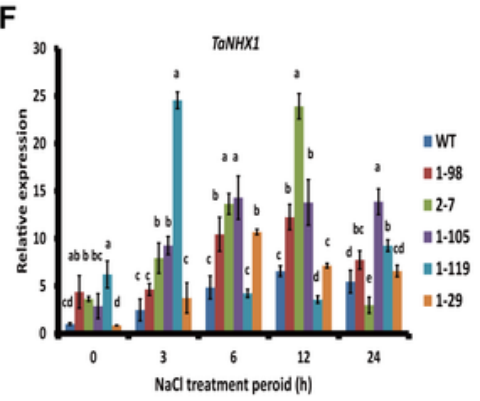

C

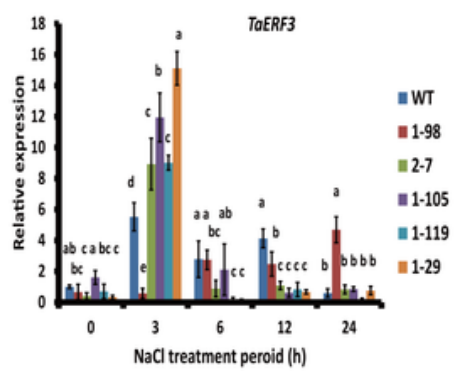

H

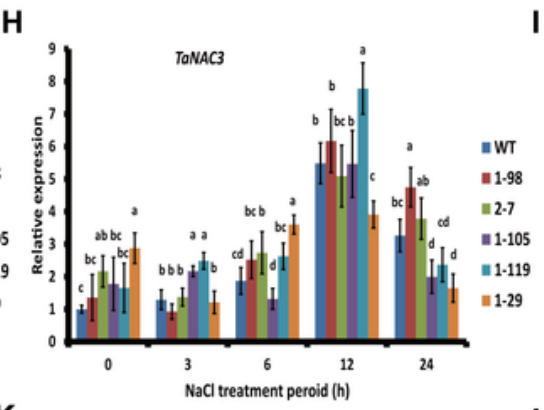

K

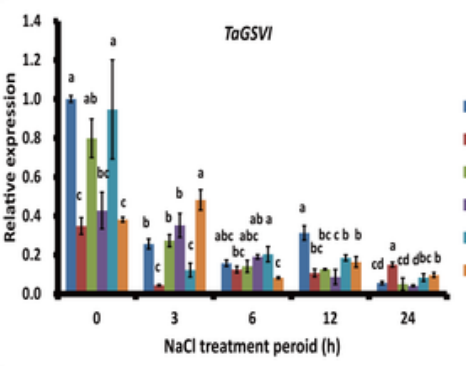

N

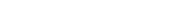

L
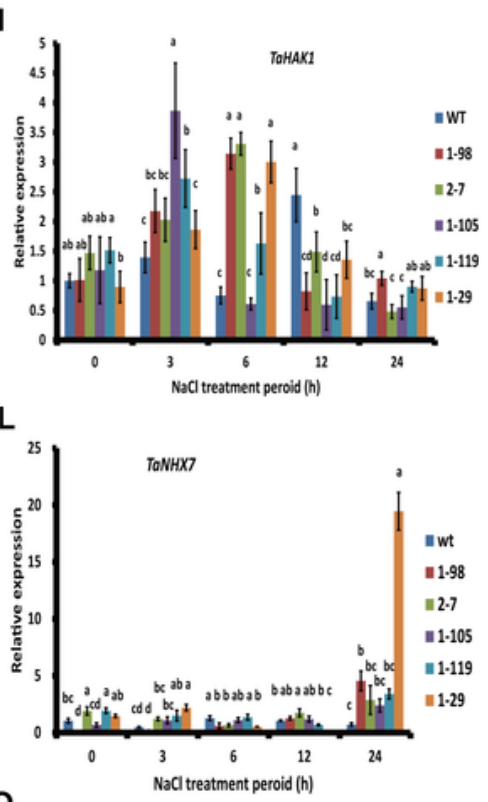

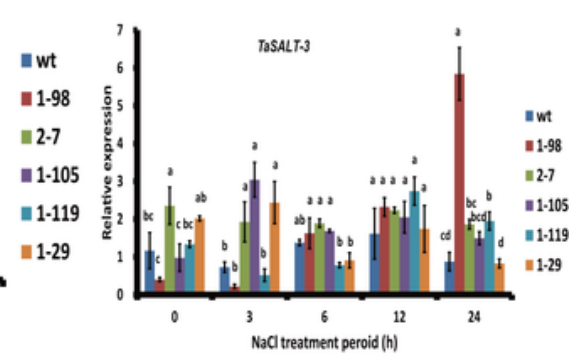

Figure 6

Expression profiles of the ethylene-related genes and salt-related genes in the wheat lines. (A - E) qRT-PCR assays for the relative expression of ethylene-related genes in different wheat plants treated with $150 \mathrm{mM}$ $\mathrm{NaCl}$ for different periods $(0-24 \mathrm{~h})$. ( $\mathrm{F}-\mathrm{O})$ qRT-PCR assays for the relative expression of the salt-related genes in different wheat plants treated with $150 \mathrm{mM} \mathrm{NaCl}$ for different periods $(0-24 \mathrm{~h})$. The statistical significance of the difference was confirmed by ANOVA at $a=0.05$ level. 
A

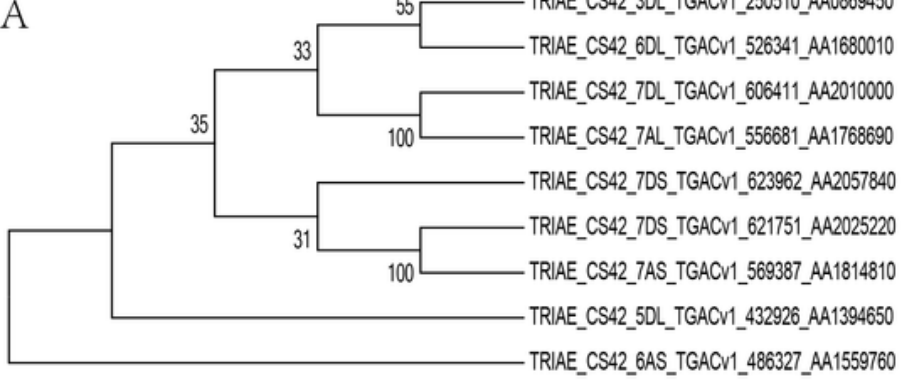

B

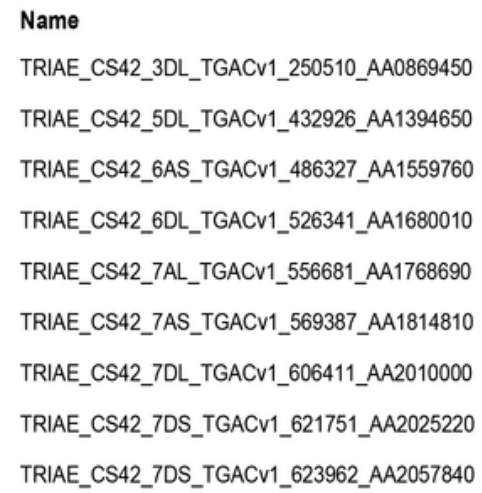

TRIAE_CS42_3DL_TGACv1_250510_AA0869450 TRIAE_CS42_5DL_TGACV1_432926_AA1394650 TRIAE_CS42_6AS_TGACv1_486327_AA1559760 TRIAE_CS42_6DL_TGACv1_526341_AA1680010 TRIAE_CS42_7AL_TGACV1_556681_AA1768690 TRIAE_CS42_7AS_TGACV1_569387_AA1814810 TRIAE_CS42_7DL_TGACv1_606411_AA2010000 TRIAE_CS42_7DS_TGACV1_621751_AA2025220 TRIAE_CS42_7DS_TGACv1_623962_AA2057840

\section{p-value Motif Locations}

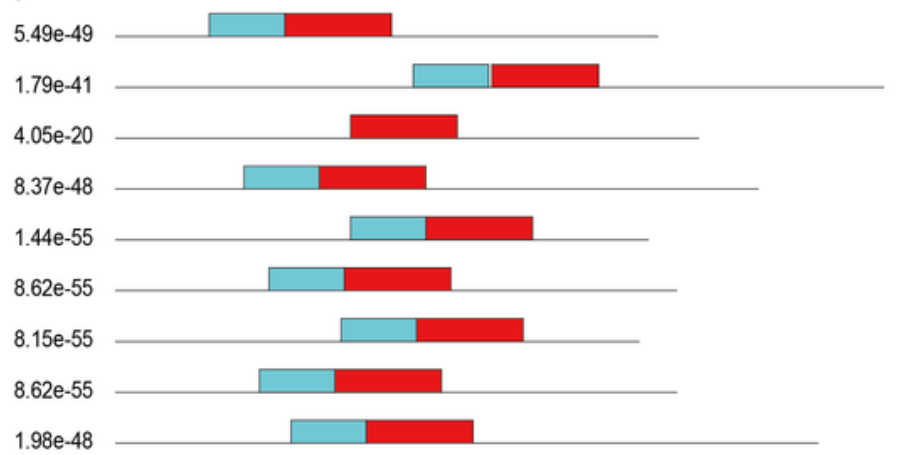

Motif Symbol Motif Consensus

1. VWLGTFDTAEEAARAYDQAAFRLRGRAARLNFPD
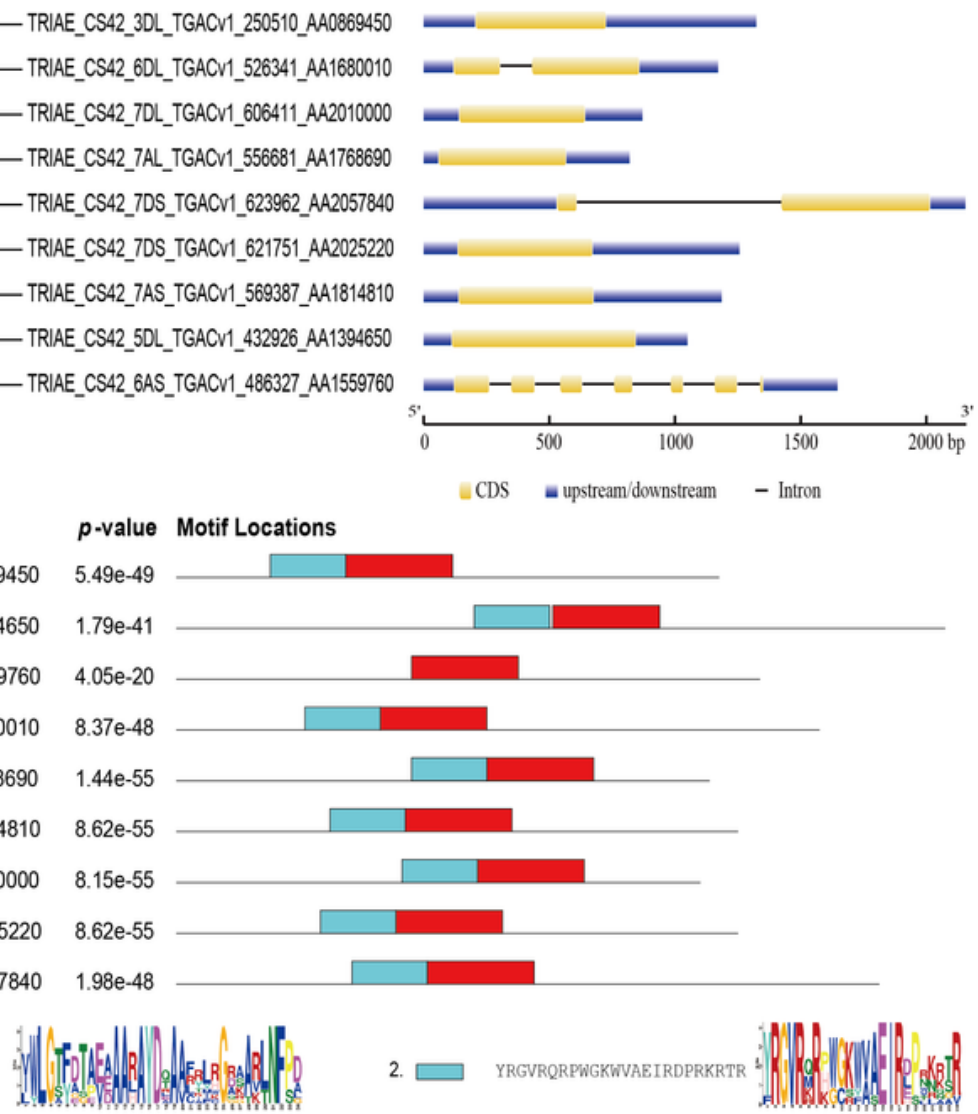

$\operatorname{cDS}$

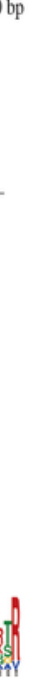

C

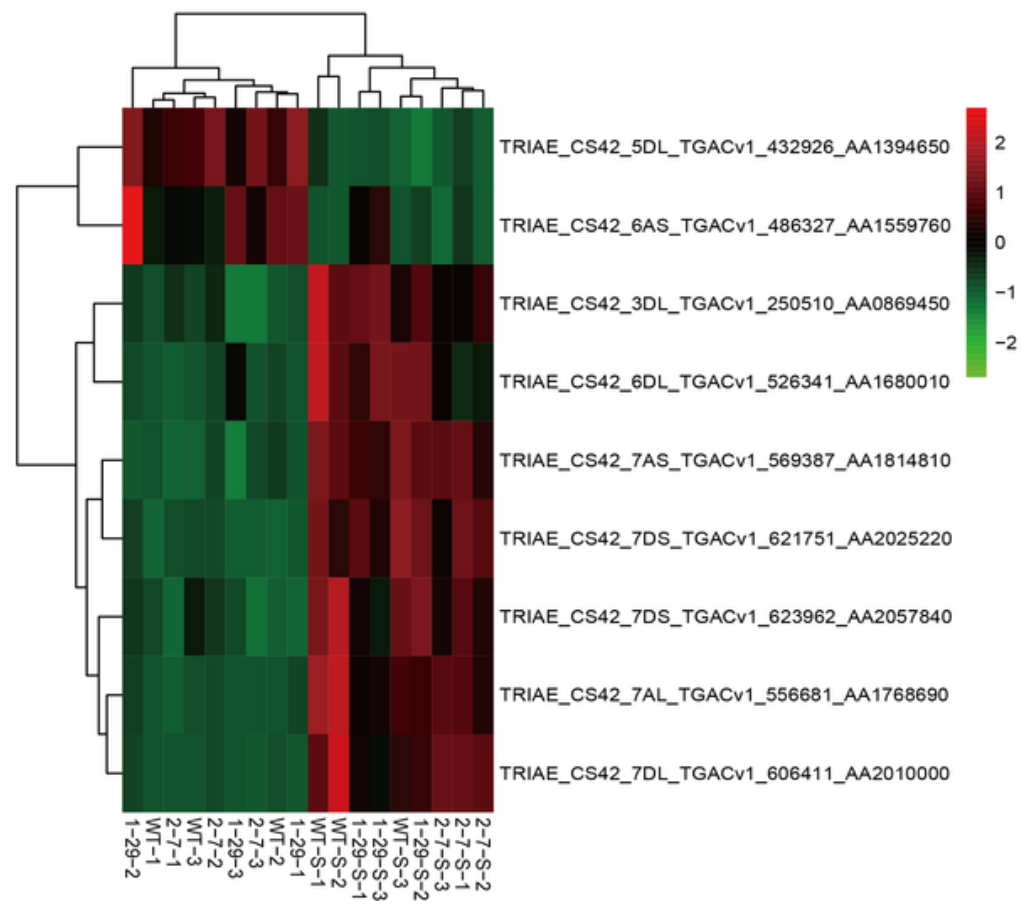

\section{Figure 7}

The wheat ERF genes in response to ethylene and salt stress. (A) Phylogenetic relationship and exonintron structure of selected ERFs. Neighbour-joining tree was constructed using MEGA Bootstrap values were indicated on the nodes of branches. The yellow boxes denoted CDS, the blue boxes indicated UTRs, and the black lines represented introns. (B) The structures and conserved domains of selected ERFs. The red boxes denoted domain 1, and the light blue boxes represented domain 2. The domain 2 and domain 1 
constituted the known GCC-box binding domain. (C) Heatmap of the expression level of selected ERFs in transcriptional sequence. The red color indicated high-expressed genes, and blue indicated low-expressed genes.

\section{Supplementary Files}

This is a list of supplementary files associated with this preprint. Click to download.

- SUPPLEMENTARYMATERIAL8.docX

- SUPPLEMENTARYMATERIAL7.docX

- SUPPLEMENTARYMATERIAL6.docx

- SUPPLEMENTARYMATERIAL5.docx

- SUPPLEMENTARYMATERIAL4.xIs

- SUPPLEMENTARYMATERIAL3.xIs

- SUPPLEMENTARYMATERIAL2.xIs

- SUPPLEMENTARYMATERIAL1.png 